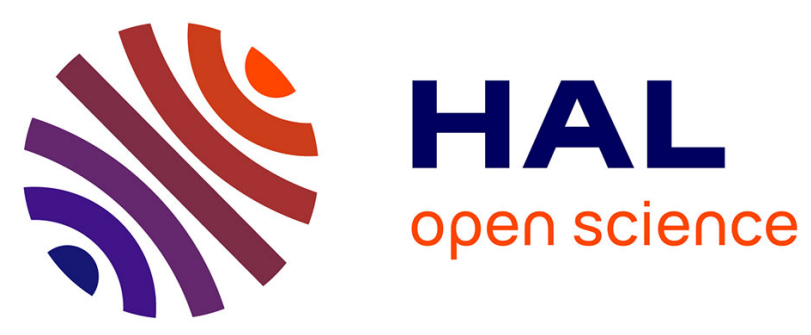

\title{
p-Toluenesulfonic acid-promoted selective functionalization of unsymmetrical arylalkynes: a regioselective access to various arylketones and heterocycles
}

Maud Jacubert, Olivier Provot, Jean-François Peyrat, Abdallah Hamze, Jean-Daniel Brion, Mouad Alami

\section{To cite this version:}

Maud Jacubert, Olivier Provot, Jean-François Peyrat, Abdallah Hamze, Jean-Daniel Brion, et al.. p-Toluenesulfonic acid-promoted selective functionalization of unsymmetrical arylalkynes: a regioselective access to various arylketones and heterocycles. Tetrahedron, 2010, 66 (21), pp.3775-3787. 10.1016/j.tet.2010.03.055 . hal-02394573

\section{HAL Id: hal-02394573 \\ https://hal.science/hal-02394573}

Submitted on 4 Dec 2019

HAL is a multi-disciplinary open access archive for the deposit and dissemination of scientific research documents, whether they are published or not. The documents may come from teaching and research institutions in France or abroad, or from public or private research centers.
L'archive ouverte pluridisciplinaire HAL, est destinée au dépôt et à la diffusion de documents scientifiques de niveau recherche, publiés ou non, émanant des établissements d'enseignement et de recherche français ou étrangers, des laboratoires publics ou privés. 


\title{
$p$-Toluenesulfonic Acid-Promoted Selective Functionalization of Unsymmetrical Arylalkynes: A Regioselective Access to various Arylketones and Heterocycles
}

\author{
Maud Jacubert, Olivier Provot, ${ }^{*}$ Jean-François Peyrat, Abdallah Hamze, Jean-Daniel Brion \\ and Mouâd Alami* \\ Univ Paris-Sud, CNRS, BioCIS, UMR 8076, Laboratoire de Chimie Thérapeutique, Faculté de Pharmacie, rue J.-B. Clément F-92296 \\ Châtenay-Malabry, France.
}

\begin{abstract}
Regioselective hydration of a wide range of internal alkynes has been afforded in high to good yields by using PTSA in EtOH. The scope of the reaction of alkynes has been delineated. Arylaliphatic alkynes and diarylalkynes were regioselectively hydrated in good to excellent yields and short reaction times when the reaction was achieved under microwave irradiation. Moreover, diarylalkynes, arylenynes as well as diaryldiynes bearing a methoxy- or a thiomethyl substituent on the ortho position underwent a regioselective 5-endo-dig-cyclization to give a variety of 2-aryl- and 2-styrylbenzofuran or benzothiphene derivatives. We believe that, this new environmentally metal-free procedure combined to microwave irradiation would be in importance in the search of green laboratory-scale synthesis. (C) 2019 Elsevier Science. All rights reserved
\end{abstract}

Keywords: Alkynes; Hydration; p-toluenesulfonic acid; Ketone; Benzofuran; Benzothiophene; Cyclization.

\section{Introduction}

The hydration of alkynes is an important tool to construct a carbon-oxygen bond, ${ }^{1}$ and provides a direct access to carbonyl compounds with a perfect atom economy. Historically, this reaction was described by Kucherov since $1881,{ }^{2}$ and required the use of mercury(II) salts. The necessity of strongly acidic conditions however, associated to the use of stoichiometric amounts of toxic mercury salts had always been an inherent problem of the process, making this reaction unsuitable for modern applications. In recent years, numerous attempts have been made to develop non-mercury alkyne hydration methodology. To this end, transition-metal complex catalysts including $\mathrm{Au},{ }^{3} \mathrm{Ru},{ }^{4} \mathrm{Ir},{ }^{5} \mathrm{Rh},{ }^{6} \mathrm{Pt}^{7}$ and $\mathrm{Pd}^{8}$ have been employed with varying degrees of success, but the use of high loading of expensive transition-metal catalysts limits the exploitation of these methods. Beside enzymatic hydration of alkynes, ${ }^{9}$ metal-free procedures using a catalytic Brønsted acid such as $\mathrm{TfOH}$ or $\mathrm{Tf}_{2} \mathrm{NH}^{10}$ or concentrated $\mathrm{H}_{2} \mathrm{SO}_{4},{ }^{11}$ or $\mathrm{HCO}_{2} \mathrm{H}^{12}$ have been reported. While these latter hydration reactions are suitable in the case of robust substrates, they are limited to terminal alkynes and are not applicable for oxygenated alkynes ${ }^{12}$ where the triple bond remained absolutely inert.

As part of our research program directed to a selective functionalization of unsymmetrically disubstituted alkynes, ${ }^{13}$ we had to investigate the tetrahydropyranylether cleavage of 1a in EtOH under $p$-toluenesulfonic acid (PTSA) conditions. ${ }^{14}$ Contrary to our expectations, the reaction did not afford $\mathbf{1 b}$. We serendipitously found that, besides the THP-ether cleavage, the internal carboncarbon triple bond of 1a was hydrated together with a concomitant etherification to give regioselectively $\mathbf{2 a}$ as the only product in a $90 \%$ yield. Under these conditions, performing the reaction from $\mathbf{1 b}$ assisted both etherification of free hydroxyl group and carbonyl formation, producing $\mathbf{2 a}$ in an excellent $94 \%$ isolated yield. One can note that in both cases, the hydration reaction is chemoselective, as it occurred exclusively on the internal carbon-carbon triple bond keeping terminal alkynes unchanged (Scheme 1).

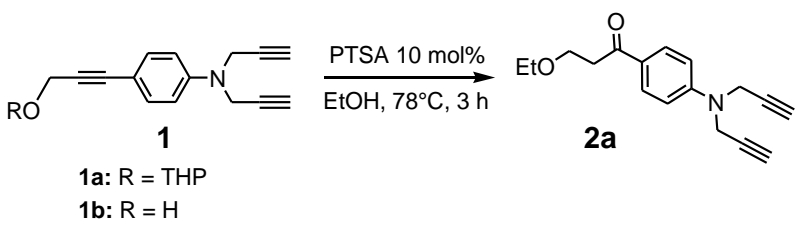

Scheme 1.

Because the discovery of metal-free alkynes hydration procedures remain as an intruiging challenge, the above observations led us to experiment the PTSA-alcohol system as a new and regioselective access to carbonyl compounds. Herein we wish to detail our results with a variety of unsymmetrical internal alkynes including, aliphatic arylalkynes or alkynols, diarylalkynes, 1,3enynes and 1,3-diynes.

\footnotetext{
* Corresponding authors. Tel.; +33 14683 5847; fax: +33 146835828 (OP); tel.; +33 14683 5887; fax: +33 14683 5828 (MA). e-mail adresses: olivier.provot@u-psud.fr (O. Provot); mouad.alami@u-psud.fr (M. Alami).
} 
Table 1. Screening of catalysts and solvents for hydration of 4-methoxyphenyl alkynols 1c-h.

\begin{tabular}{|c|c|c|c|c|c|c|c|}
\hline entry & alkyne $\mathbf{1}$ & & $\begin{array}{c}\text { Catalyst } \\
(20 \mathrm{~mol} \%)\end{array}$ & $\begin{array}{l}\mathrm{ROH} \\
\text { (time) }\end{array}$ & & ketone 2 & Yield $^{\mathrm{a}}(\%)$ \\
\hline 1 & & $1 \mathrm{c}$ & PTSA & $\begin{array}{c}\mathrm{EtOH} \\
(5 \mathrm{~h})\end{array}$ & $2 \mathrm{~b}$ & & 94 \\
\hline 2 & $"$ & $1 \mathrm{c}$ & $\mathrm{MeSO}_{3} \mathrm{H}$ & $\begin{array}{c}\mathrm{EtOH} \\
(5 \mathrm{~h})\end{array}$ & $2 b$ & $"$ & 85 \\
\hline 3 & $"$ & $1 c$ & $\mathrm{CF}_{3} \mathrm{SO}_{3} \mathrm{H}^{\mathrm{b}}$ & $\begin{array}{c}\mathrm{EtOH} \\
(5 \mathrm{~h})\end{array}$ & $3 \mathbf{a}$ & & 60 \\
\hline 4 & $"$ & $1 \mathrm{c}$ & PTSA & $\begin{array}{c}\mathrm{MeOH} \\
(6 \mathrm{~h})\end{array}$ & $2 c$ & & 98 \\
\hline 5 & " & $1 c$ & PTSA & $\begin{array}{c}i \mathrm{PrOH} \\
(7 \mathrm{~h})\end{array}$ & 2d & & 66 \\
\hline 6 & $"$ & $1 \mathrm{c}$ & PTSA & $\begin{array}{c}t \mathrm{BuOH} \\
(24 \mathrm{~h})\end{array}$ & & - & 0 \\
\hline 7 & $"$ & $1 \mathrm{c}$ & PTSA & $\underset{(17 \mathrm{~h})}{\mathrm{HO}}$ & $2 e$ & & 33 \\
\hline 8 & $"$ & $1 \mathrm{c}$ & PTSA & $\begin{array}{l}\mathrm{H}_{2} \mathrm{O} \\
(10 \mathrm{~h})\end{array}$ & $2 f$ & & 79 \\
\hline 9 & $"$ & $1 c$ & PTSA & $\begin{array}{c}\mathrm{CD}_{3} \mathrm{OD} \\
(5 \mathrm{~h})\end{array}$ & $D-2 c$ & & 96 \\
\hline 11 & & 1d & PTSA & $\begin{array}{c}\mathrm{EtOH} \\
(8 \mathrm{~h})\end{array}$ & $2 b$ & & 90 \\
\hline 12 & & 1e & PTSA & $\begin{array}{l}\text { EtOH } \\
(24 \mathrm{~h})\end{array}$ & $2 \mathrm{~g}$ & & 85 \\
\hline 13 & & 1f & PTSA & $\begin{array}{l}\text { EtOH } \\
(36 \mathrm{~h})\end{array}$ & $2 \mathrm{~h}$ & & 90 \\
\hline 14 & & $1 \mathrm{~g}$ & PTSA & $\begin{array}{l}\text { EtOH } \\
(24 \mathrm{~h})\end{array}$ & $2 \mathrm{i}$ & & 91 \\
\hline 15 & & $1 \mathrm{~h}$ & PTSA & $\begin{array}{l}\text { EtOH } \\
(10 \mathrm{~h})\end{array}$ & $3 b$ & & 73 \\
\hline
\end{tabular}

${ }^{\text {a }}$ Isolated yield

b $30 \mathrm{~mol} \%$ of catalyst were used.

\section{Results and Discussion}

\subsection{Hydration-etherification of aliphatic arylalkynols ${ }^{15}$}

The required alkynols $\mathbf{1 c}-\mathbf{h}$ were readily prepared by Sonogashira-Linstrumelle (S-L) coupling reactions. ${ }^{16}$ The reactivity of model alkyne 1c was first examined in the presence of different Brønsted acids and solvents; the results are listed in Table 1 . Thus, reaction of $1 \mathrm{c}$ with PTSA or $\mathrm{MeSO}_{3} \mathrm{H}(20 \mathrm{~mol} \%)$ in refluxing EtOH for $5 \mathrm{~h}$ provides $\mathbf{2 b}$ resulting from a regioselective hydration and etherification of the free alcohol (entries 1 and 2). It is noteworthy that enone $\mathbf{3 a}$ was formed when $\mathrm{CF}_{3} \mathrm{SO}_{3} \mathrm{H}$ was used as catalyst (entry 3,60\%). All of the other tested catalytic systems $\left(\mathrm{H}_{2} \mathrm{SO}_{4}, \mathrm{CF}_{3} \mathrm{COOH}, \mathrm{HCO}_{2} \mathrm{H}\right)$ were unsuccessful, and in better cases, yields in hydration products 2 were not satisfactory (e.g.; $\mathrm{HCOOH,} \mathrm{50 \% ,} \mathrm{data}$ not shown). The influence of the solvent was next examined with 1c and PTSA (entries 4-9). The hydration and etherification of $1 c$ occurred efficiently in refluxing $\mathrm{MeOH}$ (entry 4) yielding 2c in $98 \%$. The process was slower and less effective in $i \mathrm{PrOH}$ or 1,2-ethyleneglycol (entries 5 and 7) whereas, in $t \mathrm{BuOH}$ no reaction occurred, even after a prolonged reaction time (entry 6). Interestingly, when the hydration was performed in refluxing water for $10 \mathrm{~h}$, the triple bond was regioselectively hydrated giving ketone $2 \mathbf{f}$ with a free alcohol function in a 79\% yield (entry 8). Carrying out the reaction with $1 c$ in $\mathrm{CD}_{3} \mathrm{OD}$ as a solvent (entry 10), besides a deuterated methyl ether formed, a quantitative deuterium incorporation on the $\alpha$-carbon of the keto group was observed yielding $\boldsymbol{D}$-2c (entry 9, 96\%). 


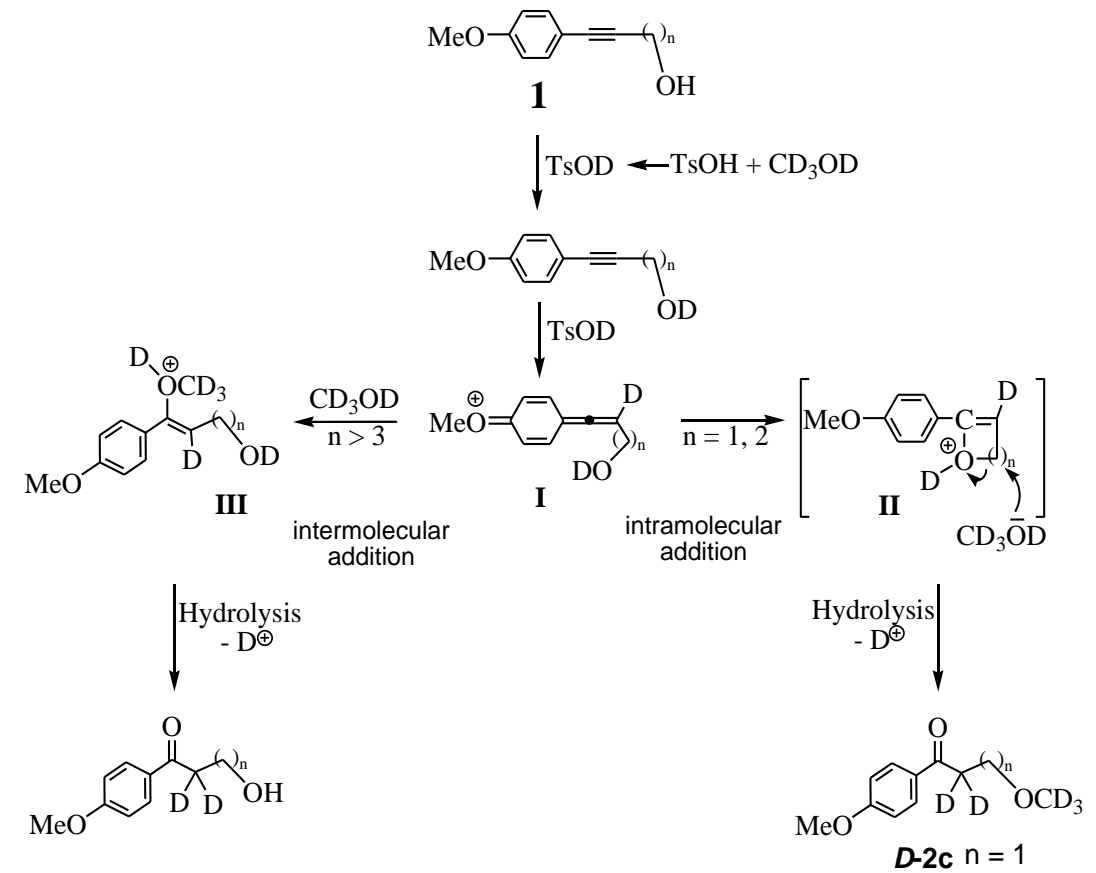

Scheme 2. Mechanism proposed for PTSA-catalyzed hydration of alkynols in $\mathrm{CD}_{3} \mathrm{OD}$

Next, the PTSA/EtOH system was used for the hydration of alkyne 1d bearing a methylether function. In this case, 1d also underwent hydration of the triple bond together with a trans-etherification reaction by the solvent resulting in a 90\% yield of the ethylether carbonyl compound $\mathbf{2 b}$ (entry 11). Finally, the influence of alkynol chains on the hydration-etherification process was investigated. As shown in entry 12 , compound 1e bearing on the aromatic ring a butyn-4-ol chain, was transformed into the expected ketone $\mathbf{2} \mathbf{g}$ in a good yield but with a prolonged reaction time $(24 \mathrm{~h})$. Similarly, hydration of alkyne $\mathbf{1 f}$ having on the aromatic nucleus a hexyn-6-ol chain was still effective (entry 13). In this case, no etherification of the hydroxyl group occurred and $\mathbf{2 h}$ with a free-alcohol function was isolated in a good $90 \%$ yield. Reaction from arylalkyne $\mathbf{1 g}$ having a secondary propargylic alcohol function provided the expected carbonyl ether $\mathbf{2 i}$ (91\%, entry 14). Under these conditions, tertiary propargyl alcohol $\mathbf{1 h}$ leads in $73 \%$ yield to the corresponding conjugated ketone $\mathbf{3 b}$, well known as the Rupe's rearrangement ${ }^{17}$ adduct (entry 15).

\subsection{Mechanism of PTSA-catalyzed hydration and etherification of arylalkynols in $\mathrm{CD}_{3} \mathrm{OD}$.}

The formation of arylketones $\mathbf{2}$ is believed to proceed initially through a deuterium exchange between $\mathrm{CD}_{3} \mathrm{OD}$ and PTSA followed by acidic deuterium activation of the triple bond as outline in Scheme 2. The intermediate species of type I should evolve according to an intra or an intermolecular addition process depending on the longer alkynol chains. With substrates having a propyn-3-ol or a butyn-4-ol chain $(\mathbf{1 c} ; n=1$ or $\mathbf{1 e} ; n=2)$ the reaction would evolved according to an intramolecular oxygen atomaddition to provide a four or a five membered cyclic oxonium intermediate II. Nucleophilic ring opening with $\mathrm{CD}_{3} \mathrm{OD}$ and subsequent hydrolysis regenerated the catalyst and produced a deuterated enol intermediate immediately rearranged to the corresponding keto tautomer $\boldsymbol{D}$-2c (entry 9, Table 1). From substrate $\mathbf{1 f}$ having longer alkynol chain $(n=4$, entry 13 , Table 1$)$, direct alcoholic media addition ${ }^{18}$ on $\mathbf{I}$ is preferred in comparison with thermodynamically disfavored 7-membered cyclic oxonium intermediate formation, keeping free the hydroxyl group. As a check on our mechanistic interpretation, we also examined hydration of a substrate 1i bearing a pentyl-5-ol chain $(n=3)$. Under the same reaction conditions, hydration of $\mathbf{1 i}$ evolved according to the two routes to give a mixture of alcohol $\mathbf{2 j}$ and ether $\mathbf{2 k}$ (Scheme 3 ).

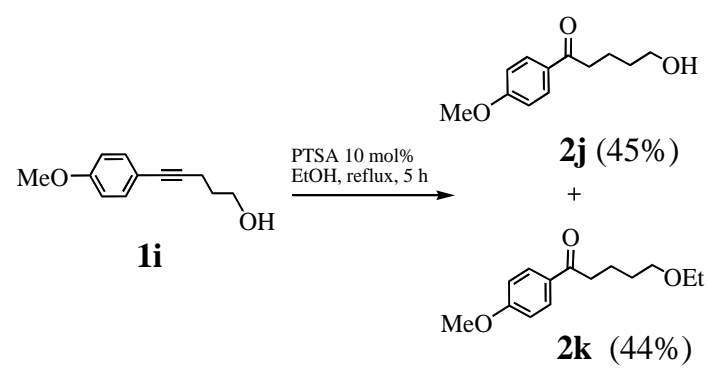

Scheme 3. Hydration of alkynol 1i

\subsection{Hydration of aliphatic arylalkynes}

The scope of this PTSA-catalyzed hydration reaction was next investigated with several arylalkyne substrates and the results of this study are summarized in Table 2. 
Table 2. PTSA catalyzed synthesis of various arylketones $\mathbf{2}$

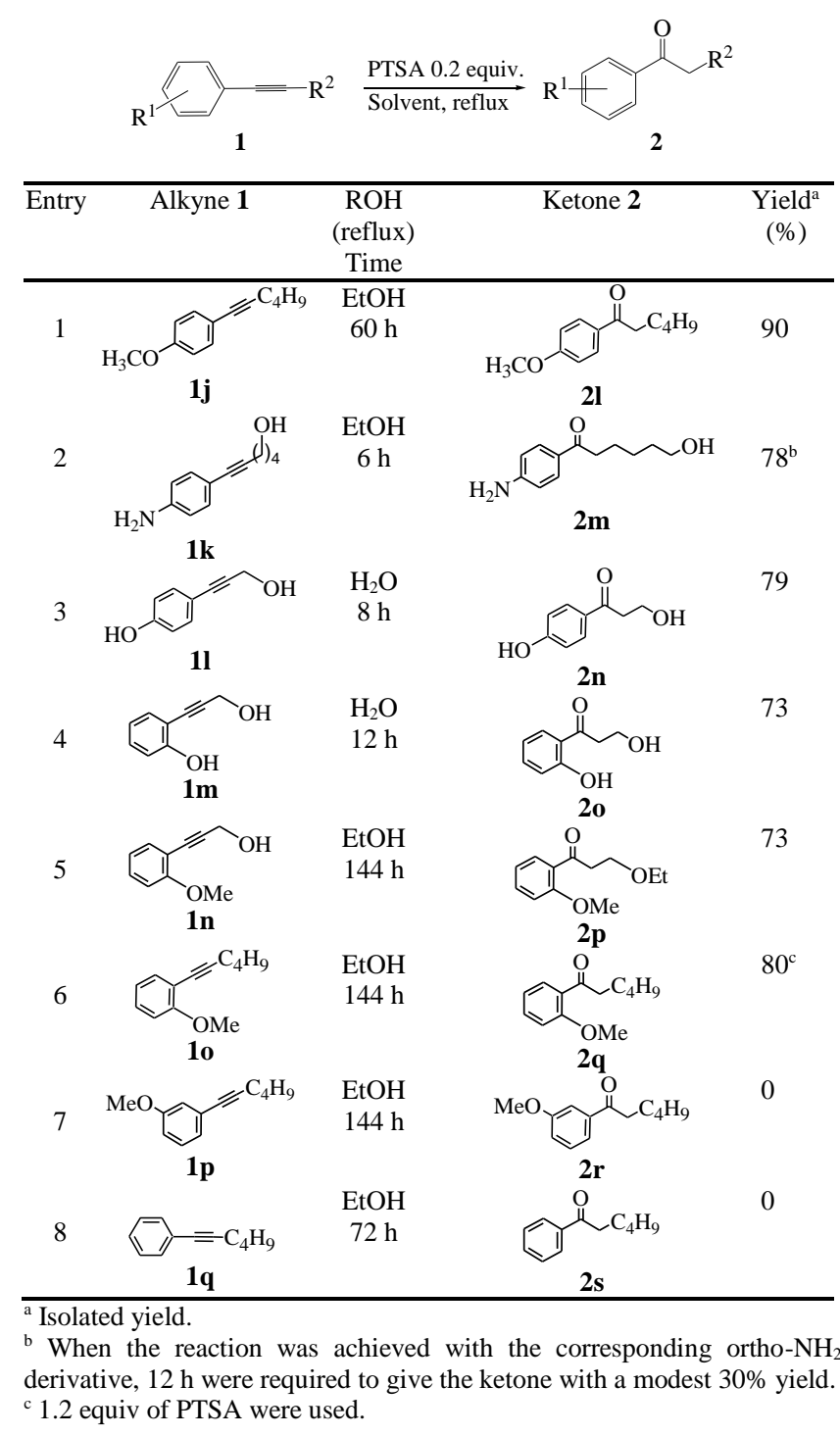

Arylalkyne $\mathbf{1 j}$ bearing a hexynyl side chain was successfully hydrated (entry $1,90 \%$ ) but $60 \mathrm{~h}$ were required for complete conversion. Next, other $p$-electron-donating groups on the aromatic ring were evaluated to assist the hydration process. As shown in entries 2 and 3 , a para $\mathrm{NH}_{2}$ and a para- $\mathrm{OH}$ substituents on the aromatic ring activated the triple bond, as suggested in the mechanism, and the corresponding ketones $\mathbf{2 m}$ and $\mathbf{2 n}$ were obtained in $\mathrm{EtOH}$ or $\mathrm{H}_{2} \mathrm{O}$ in good yields. On switching the electron-donating $\mathrm{OH}$-group from the para to the ortho position on the aromatic nucleus, the hydration in $\mathrm{H}_{2} \mathrm{O}$ was still efficient but required a prolonged reaction time (compare entries 3 and 4, Table 2). In refluxing $\mathrm{EtOH}$, ortho anisole alkyne derivatives $1 \mathbf{n}$ and $\mathbf{1 o}$ reacted smoothly to give arylketones 2p and 2q. In this latter case, 1.2 equiv of PTSA were necessary to achieve the complete hydration of the alkyne substrate 10 (entry 6). However, in agreement with the proposed mechanism, meta anisole derivative $\mathbf{1 p}$ as well as non-substituted arylalkyne 1q did not react under the above experimental conditions, even with stoechiometric amount of PTSA and prolonged reaction times. In these cases, starting arylalkynes were recovered unchanged (entries 7 and 8 ). These findings were illustrated with the selective hydration of alkyne 1r having two carbon-cabon triple bonds. As expected, besides the etherification of the free alcohol function by the solvent, only the activated triple bond underwent the hydration reaction leading to $2 \mathbf{t}$ in $76 \%$ yield (Scheme 4).

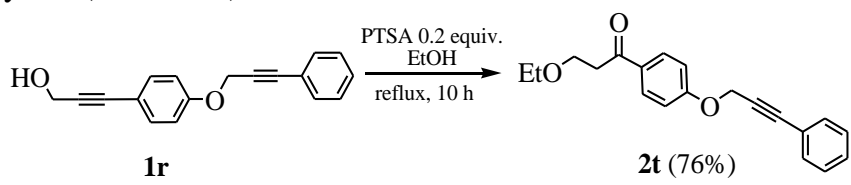

Scheme 4. Selectivity in triple bond hydration of $\mathbf{1 r}$

To circumvent the low reactivity of ortho-substituted arylalkynes and the lack of reactivity of non-substituted arylalkynes as well as meta-substituted derivatives, we envisioned to use microwave irradiation (MWI) ${ }^{19}$ that could enhance this process and expand the substrate scope. Moreover, with cooperative para-substituted arylalkynes, the use of MWI should accelerate the rate of this hydration reaction.

Initial efforts focused on optimizing microwave conditions for the formation of $\mathbf{2 s}$ from 1q. After screening the quantity of PTSA, temperature and reaction time, we found that complete conversion of $1 \mathbf{q}$ required the use of PTSA (1 equiv) in $\mathrm{EtOH}$, for $30 \mathrm{~min}$ at $170{ }^{\circ} \mathrm{C}$ under MWI to give 2 s in $89 \%$ yield (entry 1 , Table 3 ). Performing the reaction at a lower temperature $\left(120^{\circ}\right.$ or $\left.150{ }^{\circ} \mathrm{C}\right)$ or using a catalytic amount of PTSA $(20 \mathrm{~mol} \%)$ resulted in lower yields of $2 \mathrm{~s}$ (15-35\%, data not shown). The optimized reaction conditions found for 1q were applied to meta substituted arylalkyne $\mathbf{1 p}$ which was transformed into $\mathbf{2 r}$ within $30 \mathrm{~min}$ in a satisfactory $60 \%$ yield (entry 2 , Table 3 ). To evaluate the significant benefit of MWI, model arylakyne 1c with a cooperative para methoxy substituent was heated in EtOH and PTSA ( 0.2 equiv.) at different temperatures. The best result was obtained when $1 \mathrm{c}$ was heated at $120{ }^{\circ} \mathrm{C}$ for 30 min under MWI (entry 3, 98\%). Similarly, we have applied this protocole to various arylalkynes. In most cases studied, hydration and etherification process successfully occurred (entries 3, 4, 6 and 7), except with secondary propargylic alcohol 1s (entry 5) where the Rupe's rearrangement predominated to give the conjugated ketone 3c. With 1,1dichloroenyne $\mathbf{1 u}$, beside the triple bond hydration, alcoholysis of the carbon-chlorine bonds occurred to produce the ketoester $2 \mathrm{v}$ in a $51 \%$ isolated yield (entry 8). Silylated arylalkyne 1v reacted well and furnished 4methoxyacetophenone $\mathbf{2 w}$ in $77 \%$ yield (entry 9). Replacing the 4-methoxy by a 4- $\mathrm{NH}_{2}$ substituent has no deleterious effect on this acidic hydration process and ketone $2 \mathbf{x}$ was obtained in a 91\% yield (entry 10) Finally hydration of ortho methoxyarylalkyne 10 successfully occurred within only $1 \mathrm{~h}$ at $170{ }^{\circ} \mathrm{C}$ (entry 11), whereas under conventional heating (entry 6 Table 2) $144 \mathrm{~h}$ were required to complete the hydration of the alkyne substrate. 
Table 3. PTSA promoted hydration of arylalkynes 1 under MWI

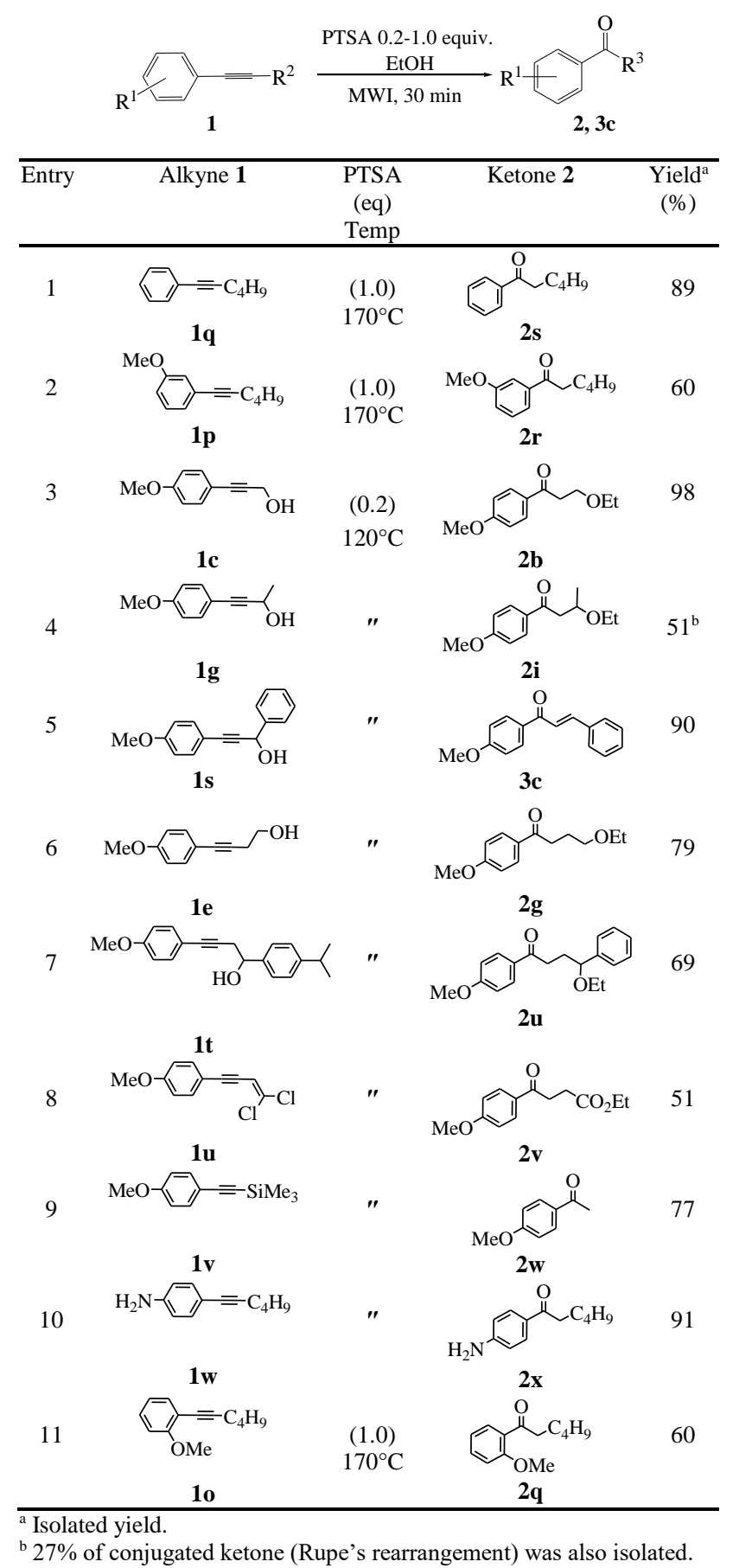

The PTSA-assisted hydration of unsymmetrical diarylalkynes 4a-f under MWI was next evaluated (Table 4, entries 1-6). By using 1.2 equiv of PTSA at $120{ }^{\circ} \mathrm{C}$ in EtOH, diarylalkyne 4a was regioselectively hydrated within 30 min (entry 1,97\%). As shown in entry 2, the presence of a bromine atom was tolerated and ketone $\mathbf{5 b}$ was regioselectively obtained in a nearly quantitative yield (entry 2, 98\%). Diarylalkynes $\mathbf{4 c}$ and $\mathbf{4 d}$, bearing respectively a methyl and a $\mathrm{NH}_{2}$-substituent on the aromatic rings reacted smoothly under the previous conditions $\left(120^{\circ} \mathrm{C}\right)$. After some trials, we were pleased to observe the total conversion of these substrates into the corresponding ketones $\mathbf{5 c}$ and $\mathbf{5 d}$ within 30 min using 1.0 equiv of PTSA under microwave heating at $150{ }^{\circ} \mathrm{C}$ (entries 3 and 4). Surprisingly, the 3,4,5-trimethoxyphenyl nucleus frequently found in many anticancer substances, ${ }^{20}$ deactivated the triple bond. In these cases, a microwave heating at $170{ }^{\circ} \mathrm{C}$ in the presence of 1 equiv of PTSA were needed to achieve a total conversion. Substrate $\mathbf{4 e}$ furnished regioselectively ketone 5e (entry 5) whereas, a mixture of ketones was obtained from alkyne 4f having methoxy substituents on both aromatic nucleus (entry 6).

Table 4. PTSA-promoted hydration of substituted diarylalkynes 4 under MWI.

(Equiv)
6

\subsection{PTSA promoted hydration of ortho-substituted arylalkynes under MWI}

Having succeeded in developing an efficient hydration process of low reactive diarylalkynes, we next examined the reaction with ortho substituted diarylalkynes under MWI at $170{ }^{\circ} \mathrm{C}$ in the presence of 1 equiv of PTSA in EtOH..$^{21}$ The results of this study are summarized in Table 5. Under the above conditions, diarylalkyne 6a bearing an ortho methoxy substituent on the aromatic ring surprisingly, provided the cyclized 2-arylbenzo[b]furan 7a with no trace of the hydration products (entry $1,76 \%$ ). This result clearly showed that the nature of the substituents attached to the triple bond has a major impact on the behavior of the reaction (cyclization versus hydration). A para methoxyphenyl ring attached to the triple bond of $\mathbf{6 a}$ favors the benzofuran formation whereas, alkyne 10 with an aliphatic chain fail to undergo cyclization but provides exclusively the hydration adduct (see entry 11, Table 3). The unique formation of $\mathbf{7 a}$ is belived to proceed according to an electrophilic activation of the alkyne carbon-carbon 
triple bond with PTSA to give hypothetic species IV or VI (Scheme 5). Subsequent intramolecular oxygen cyclization

Table 5. PTSA-promoted cyclization of ortho substituted diarylalkynes 6 in EtOH under MWI.

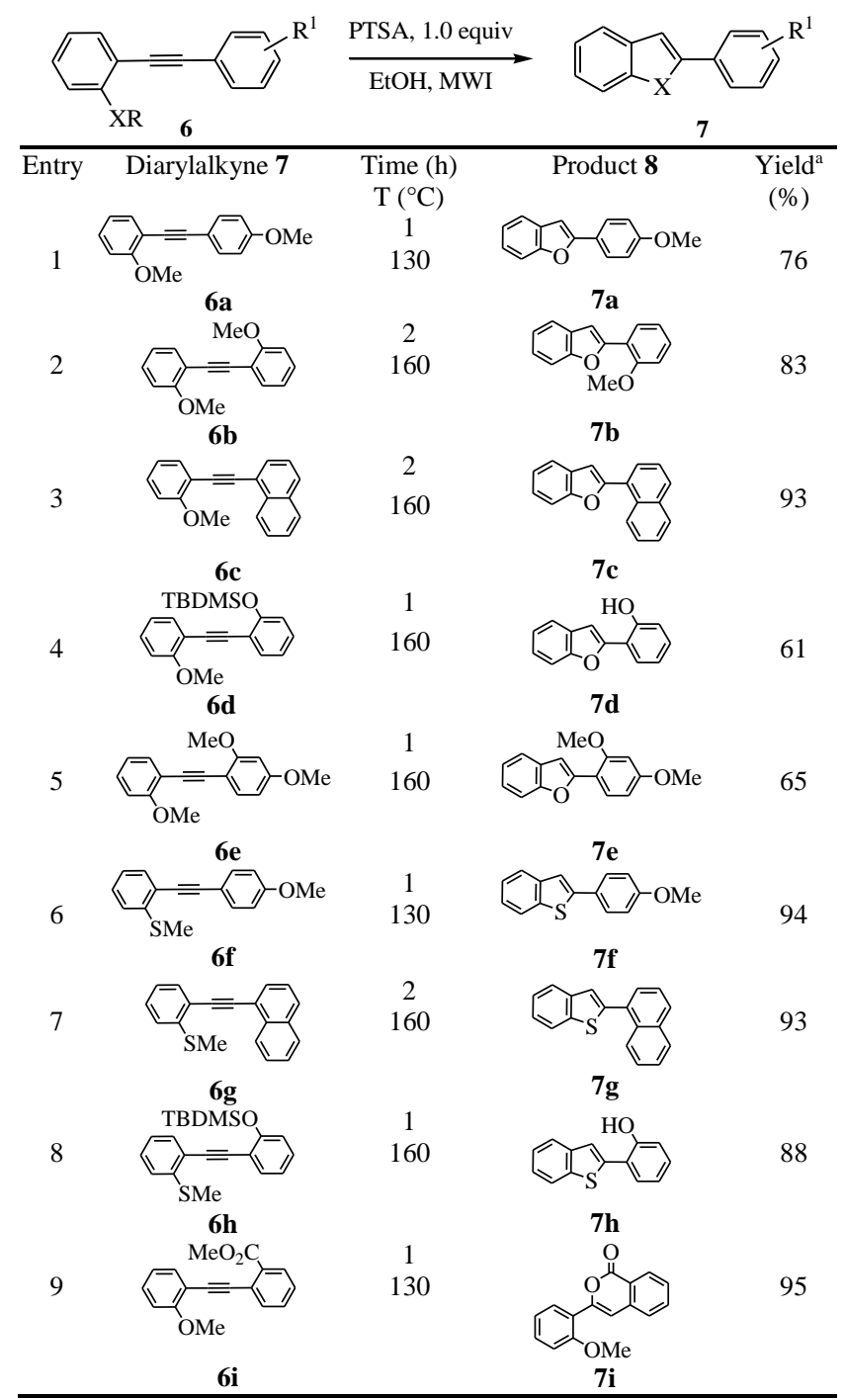

${ }^{\mathrm{a}}$ Isolated yield. from IV would form the furan nucleus of type $\mathbf{V}$ which evolves into 7a via a dealkylation step by the solvent. According to this hypothesis, the intermolecular ethanol addition from species VI is disfavored.

The scope and limitation of this cyclization was next studied with a variety of ortho substituted diarylalkynes. Substrates 6b-e bearing in the ortho position a methoxy substituent were successfully transformed into the corresponding benzofuran derivatives $\mathbf{7 b - e}$ in good yields (entries 2-5). One can note that a regioselective cyclization occurred from 6e to give a single benzofuran product 7e although alkyne substrate contains two ortho methoxy substituents (entry 5). We believe that the aromatic ring with two methoxy groups increase the electron density on the distal end of the triple bond. Subsequent electrophilic attack occurred with the ortho methoxy group of the other aromatic ring. This protocole was applied to ortho(alkynyl)thioanisole derivatives 6f-h. As expected, in $\mathrm{EtOH}$, the reaction provided the benzothiophenes $\mathbf{7 f - h}$ in good to excellent yields (88 to $94 \%$, entries 6-8). It should be noted, that this metal free procedure which proceeds under environmentally friendly conditions is amenable for large scale synthesis of 2-arylbenzofuran and 2arylbenzothiphene derivatives which are prevalent in many compounds of biological interest. ${ }^{22}$ The reaction with substrate 6i bearing both an ortho electron-donating methoxy and an electron-withdrawing methoxycarbonyl substituent was also examined. The cyclization proceeded with high selectivity and isocoumarin $7 \mathbf{i}$ was obtained as the sole product ${ }^{23}(95 \%$, entry 9$)$. This result corroborates our mechanism hypothesis as the methoxy substituent regioselectively directed the activation of the triple bond thus allowing the electrophilic cyclization to proceed by the ortho methoxycarbonyl group.

\subsection{PTSA promoted hydration of enynes 8 and diynes 9 under MWI}

In addition to the above substrates, 1,3-enynes and 1,3diynes were subjected to the previously established and already optimized electrophilic cyclization (Table 6). Enyne 8a was readily prepared by $\mathrm{Pd} / \mathrm{Cu}$-catalyzed coupling ${ }^{16 \mathrm{~b}}$ of 2-methoxyphenyl acetylene with 1-bromo-2methylpropene in a $72 \%$ yield.

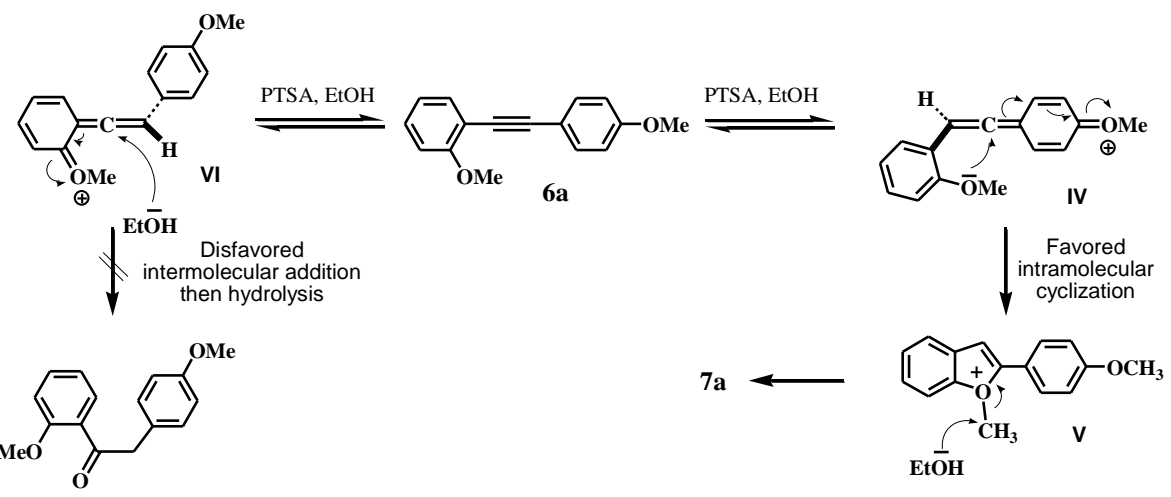

Scheme 5. Mechanism proposed for the formation of 7a. 
Table 6. Reaction of enynes 8 and diynes 9 with PTSA in EtOH under MWI. ${ }^{\mathrm{a}}$

Entry

a Reaction conditions: 8 or 9 (0.2 mmol); PTSA (0.2 mmol); EtOH (2 mL); $160^{\circ} \mathrm{C} ; 2 \mathrm{~h}$.

${ }^{\mathrm{b}}$ Isolated yield.

${ }^{\mathrm{c}}$ Reaction time $0.5 \mathrm{~h}$.

d $24 \%$ of 2-(benzofuran-2-yl)-1-2-methoxyphenyl)ethanone were also isolated.

${ }^{\mathrm{e}}$ Unoptimized yield.

${ }^{\mathrm{f}}$ Mixture of unidentified products.

For enynes $\mathbf{8 b}-\mathbf{e}$, they were obtained in good yields (See Experimental section) from iron ${ }^{24}$ or palladium ${ }^{25}$ catalyzed reaction of $(E)$-chloroenynes ${ }^{16}$ and Grignard reagents. The desired diynes 9a-e were synthesized by the coupling of 1iodoethynyl-2-methoxybenzene with terminal arylalkynes in good yields (64 to $81 \%){ }^{26}$

The PTSA protocole for the cyclization of ortho substituted arylalkynes $\mathbf{6}$ was then applied to enynes $\mathbf{8}$ and diynes $\mathbf{9 .}$ The results of this study are reported in Table 5. Reaction of enyne 8a was carried out with PTSA (1 equiv) under MWI in EtOH and gave 2-alkenylbenzofuran 10a in a satisfactory $72 \%$ yield (entry 1 ). Similarly, the reaction of (E)-enynes 8b-e gave also the corresponding 2-substituted benzofuran derivatives 10b-e with comparable yields. All the compounds synthesized were characterized by the $E$ double bond geometry as judged by ${ }^{1} \mathrm{H}$ NMR analysis (entries 2-5). The present approach seems undoubtedly promising because it allows a quick entry to a variety of 2alkenylbenzofurans of biological interest. ${ }^{27}$ The reaction was then applied to 1,3-diynes 9. Compounds 9a and 9b were readily transformed into the corresponding bisbenzofuran 11a and 2-(benzothiophenyl) benzofuran derivative 11b (entries 6 and 7). One can note that no reaction occurred when heating $9 \mathrm{a}$ at $160{ }^{\circ} \mathrm{C}$ under MWI in acidic solvents (e.g.; AcOH, HCOOH, TFA). Starting material 9a was recovered totally unchanged (data not shown). 1,3-Diyne 9c bearing a 4-methoxy substituent on one of the aromatic rings was next evaluated (entry 8). As expected, the cyclization-process occurred together with the regioselective hydration of the second carbon-carbon triple bond to furnish 11c. With a less electron-donating 2naphtyl substituent attached to the triple bond (entry 9), the process was less efficient and benzofuran 11d was isolated with a modest $28 \%$ yield. Finally, when carrying out the reaction with diyne 9e, bearing both a methoxy and carbomethoxy substituents on the ortho position of the aromatic rings, no traces of benzofuran or isocoumarin derivatives were detected.

\section{Conclusion.}

In summary, the use of PTSA in alcoholic media is a simple and efficient protocole that is easy to carry out. It appears to be predictable, regardless of the diverse array of starting materials that can be employed. Electron-rich aliphatic arylalkynes are regioselectively hydrated using a catalytic amount of PTSA in refluxing EtOH to provide carbonyl compounds. The substrate scope of this friendly procedure was successfully extended to non cooperative alkynes when carrying out the reaction under microwave irradiation. Moreover, unsymmetrical diarylalkynes bearing various functional groups on the aromatic nucleus were regioselectively and rapidely hydrated in good to excellent yields. Under the optimized conditions, diarylalkynes bearing on the ortho position a methoxy- or a thiomethyl substituent allowed an electrophilic cyclization to produce a variety of benzofuran and benzothiophene derivatives. Similarly, ortho-methoxy arylenynes were successfully transformed into the corresponding 2-alkenylbenzofurans in good yields. Although, this procedure requires high temperature (from $80^{\circ} \mathrm{C}$ to $170^{\circ} \mathrm{C}$ ), it involves non-toxic PTSA, EtOH as the solvent, short reaction times, high to excellent yields, low cost and with minimum waste. All of these advantages are in a total agreement with the green chemistry philosophy.

\section{Experimental}

\subsection{Instrumentation}

The compounds were all identified by usual physical methods, i.e. ${ }^{1} \mathrm{H}-\mathrm{NMR},{ }^{13} \mathrm{C}-\mathrm{NMR}$, IR and elemental analysis. ${ }^{1} \mathrm{H}$ and ${ }^{13} \mathrm{C}$ NMR spectra were measured in $\mathrm{CDCl}_{3}$ with a Bruker Avance $300(300 \mathrm{MHz}) .{ }^{1} \mathrm{H}$ chemical shifts are reported in $\mathrm{ppm}$ from an internal standard TMS or of 
residual chloroform (7.27 ppm). The following abreviation are used: $\mathrm{m}$ (multiplet), s (singlet), d (doublet), t (triplet), $\mathrm{dd}$ (doublet of doublet). ${ }^{13} \mathrm{C}$ chemical shifts are reported in ppm from the central peak of deuteriochloroform (77.14). IR spectra were measured on a Bruker Vector 22 spectrophotometer (neat, $\mathrm{cm}^{-1}$ ). Mass spectra were obtained with a Esquire LC Bruker spectrometer. Elemental analyses were performed with a Perkin-Elmer 240 analyser. Analytical TLC were performed on Merck precoated silica gel $60 \mathrm{~F}$ plates. Merck silica gel 60 (230-400 mesh) was used for column chromatography. Melting points (m.p.) were recorded on a Büchi B-450 apparatus and were uncorrected.

\subsection{Preparation of arylalkynes}

$N, N$-di(prop-2-ynyl)-4-(3-(tetrahydro-2H-pyran-2-yloxy)prop -1-ynyl)benzenamine 1a

To a stirred solution of 4-iodo-aniline $(2.19 \mathrm{~g}, 10 \mathrm{mmol})$ in THF (20 mL) under an argon atmosphere was added, CuI (190 mg, $1.0 \mathrm{mmol}$ ), $\mathrm{PdCl}_{2}\left(\mathrm{PPh}_{3}\right)_{2}$ (350 mg, $0.5 \mathrm{mmol}$ ), $\mathrm{Et}_{2} \mathrm{NH}(7.3 \mathrm{~g}, 100 \mathrm{mmol})$ and 2-(prop-2-ynyloxy)tetrahydro- $2 H$-pyran $(16.8 \mathrm{~g}, 12 \mathrm{mmol})$. The stirred mixture was stirred for a $5 \mathrm{~h}$ at room temperature and ether $(30 \mathrm{~mL})$ was added. After filtration on a pad of celite, the organic layer was washed with $\mathrm{H}_{2} \mathrm{O}$. After extraction and concentration to dryness, the crude mixture was dissolved in DMF $(30 \mathrm{~mL})$. Then, propargyl bromide $(3 \mathrm{~mL} ; 27.6$ mmol; $80 \%$ in toluene), $\mathrm{K}_{2} \mathrm{CO}_{3}(3.24 \mathrm{~g})$, $\mathrm{KI}(0.3 \mathrm{~g})$ were added to the mixture which was stirred at $50^{\circ} \mathrm{C}$ for $15 \mathrm{~h}$. After cooling, the crude mixture was diluted in diethylether $(30 \mathrm{~mL})$, filtered. The organic layer was washed with $\mathrm{H}_{2} \mathrm{O}(3 \times 10 \mathrm{~mL})$, dried with $\mathrm{MgSO}_{4}$ and evaporated to dryness. Purification by flash chromatography afforded 1a $(675 \mathrm{mg}, 2.22 \mathrm{mmol}$ calculated from 4-iodoaniline).

Yield : $22 \%$.

IR $\left(\mathrm{cm}^{-1}\right)$ 3287, 2942, 1606, 1514, 1340.

${ }^{1} \mathbf{H}$ NMR $\left(\mathrm{CDCl}_{3}, 300 \mathrm{MHz}, 298 \mathrm{~K}\right): \delta \mathrm{ppm}$ 1.40-1.90 (m, $6 \mathrm{H}), 2.25(\mathrm{t}, 2 \mathrm{H}, J=2.3 \mathrm{~Hz}), 3.50-3.90(\mathrm{~m}, 2 \mathrm{H}), 4.11(\mathrm{~d}$, $4 \mathrm{H}, J=2.3 \mathrm{~Hz}), 4.47(\mathrm{~d}, 2 \mathrm{H}, J=3.3 \mathrm{~Hz}), 4.89$ (t, 1H, $J=$ $3.3 \mathrm{~Hz}), 6.84(\mathrm{~d}, 2 \mathrm{H}, J=9.0 \mathrm{~Hz}), 7.37(\mathrm{~d}, 2 \mathrm{H}, J=9.0 \mathrm{~Hz})$.

${ }^{13} \mathbf{C}$ NMR $\left(\mathrm{CDCl}_{3}, 75 \mathrm{MHz}, 298 \mathrm{~K}\right)$ : $\delta$ ppm 18.9, 25.2, $30.1,40.0$ (2), 54.7, 61.8, 72.7 (2), 78.7 (2), 83.4, 85.9, 96.5, 112.9, 114.4 (2), 132.8 (2), 147.2.

Anal. Calcd for $\mathrm{C}_{20} \mathrm{H}_{21} \mathrm{NO}_{2}$ : C, 78.15; H, 6.89; N, 4.56 Found C, 78.01; H, 6.71; N, 4.49.

6-(4-Aminophenyl)hex-5-yn-1-ol 1k

TEA ( 1.5 equiv) was used in place of $\mathrm{Et}_{2} \mathrm{NH}$.

Yield : $45 \%$.

IR $\left(\mathrm{cm}^{-1}\right) 3367,2936,1608,1513,1431,1288,1176,1059$, 829.

${ }^{1} \mathbf{H}$ NMR $\left(\mathrm{CDCl}_{3}, 300 \mathrm{MHz}, 298 \mathrm{~K}\right): \delta \mathrm{ppm} 1.50-1.85(\mathrm{~m}$, $4 \mathrm{H}), 2.40(\mathrm{t}, 2 \mathrm{H}, J=5.9 \mathrm{~Hz}), 3.70(\mathrm{t}, 2 \mathrm{H}, J=5.2 \mathrm{~Hz}), 6.58$ $(\mathrm{d}, 2 \mathrm{H}, J=6.7 \mathrm{~Hz}), 7.19(\mathrm{~d}, 2 \mathrm{H}, J=6.7 \mathrm{~Hz})$.
${ }^{13} \mathrm{C}$ NMR $\left(\mathrm{CDCl}_{3}, 75 \mathrm{MHz}, 298 \mathrm{~K}\right): \delta \mathrm{ppm} \mathrm{19.2,} \mathrm{25.2,}$ 31.9, 62.5, 81.4, 87.2, 114.7 (2), 132.7 (2), 145.9 (one $\mathrm{C}$ missing).

Anal. Calcd for $\mathrm{C}_{12} \mathrm{H}_{15} \mathrm{NO}$ : C, 76.16; H, 7.99; N, 7.40 Found C, 76.05; H, 8.15; N, 7.19.

\section{2-(3-Hydroxyprop-1-ynyl)phenol 1m}

1m was prepared from the coupling of tert-butyl(2iodophenoxy)dimethylsilane with 1-propyn-3-ol as above and the crude mixture was desilylated using TBAF (5 equiv) in THF at room temperature.

Yield : $59 \%$ (for the 2 steps).

IR $\left(\mathrm{cm}^{-1}\right)$ 3290, 2938, 1605, 1510.

${ }^{1} \mathbf{H}$ NMR $\left(\mathrm{CDCl}_{3}, 300 \mathrm{MHz}, 298 \mathrm{~K}\right): \delta \mathrm{ppm} 4.70-4.90(\mathrm{bs}$, $3 \mathrm{H}), 7.01-7.23(\mathrm{~m}, 2 \mathrm{H}), 7.45(\mathrm{td}, 1 \mathrm{H}, J=7.8 \mathrm{~Hz}, J=1.3$ $\mathrm{Hz}), 7.51$ (dd, $1 \mathrm{H}, J=7.8 \mathrm{~Hz}, J=1.2 \mathrm{~Hz})$.

${ }^{13} \mathrm{C}$ NMR $\left(\mathrm{CDCl}_{3}, 75 \mathrm{MHz}, 298 \mathrm{~K}\right): \delta \mathrm{ppm} 50.4,79.9$, 93.3, 109.9, 115.3, 119.5, 129.8, 132.5, 157.9.

Anal. Calcd for $\mathrm{C}_{9} \mathrm{H}_{8} \mathrm{O}_{2}$ : C, 72.96; H, 5.44. Found C, 72.87; H, 5.34.

1-(4-isoPropylphenyl)-4-(4-methoxyphenyl)but-3-yn-1-ol 1t Yield : $88 \%$. $\mathrm{mp} 72-73^{\circ} \mathrm{C}$.

IR $\left(\mathrm{cm}^{-1}\right) 3454,2967,1607,1508,1283,1242,1175,1065$, 1037, 1016, 830 .

${ }^{1} \mathbf{H}$ NMR $\left(\mathrm{CDCl}_{3}, 300 \mathrm{MHz}, 298 \mathrm{~K}\right): \delta \mathrm{ppm} 1.24(\mathrm{~d}, 6 \mathrm{H}, J$ $=6.9 \mathrm{~Hz}), 2.42(\mathrm{~d}, 1 \mathrm{H}, J=3.3 \mathrm{~Hz}), 2.82(\mathrm{~d}, 2 \mathrm{H}, J=6.3$ $\mathrm{Hz}), 2.91(\mathrm{q}, 1 \mathrm{H}, J=6.9 \mathrm{~Hz}), 3.79(\mathrm{~s}, 3 \mathrm{H}), 4.88-4.93(\mathrm{~m}$, $1 \mathrm{H}), 6.81(\mathrm{~d}, 2 \mathrm{H}, J=8.5 \mathrm{~Hz}), 7.22(\mathrm{~d}, 2 \mathrm{H}, J=8.1 \mathrm{~Hz}), 7.32$ $(\mathrm{d}, 2 \mathrm{H}, J=8.5 \mathrm{~Hz}), 7.35(\mathrm{~d}, 2 \mathrm{H}, J=8.1 \mathrm{~Hz})$.

${ }^{13} \mathrm{C}$ NMR $\left(\mathrm{CDCl}_{3}, 75 \mathrm{MHz}, 298 \mathrm{~K}\right): \delta \mathrm{ppm} \mathrm{24.0,} \mathrm{30.6,}$ $33.8,55.3,72.5,82.9,84.6,113.7,115.4,125.8,126.5$, 133.0, 140.1, 148.6, 159.3.

MS (ESI+) $m / z=317[\mathrm{M}+\mathrm{Na}]^{+}$

Anal. Calcd for $\mathrm{C}_{20} \mathrm{H}_{22} \mathrm{O}_{2}$ : C, 81.60; H, 7.53. Found C, 81.47; H, 7.31.

\section{4-(Hex-1-ynyl)benzenamine 1w}

Yield : $65 \%$.

IR $\left(\mathrm{cm}^{-1}\right)$ 3374, 2957, 2931, 1620, 1512, 828.

${ }^{1} \mathbf{H}$ NMR $\left(\mathrm{CDCl}_{3}, 300 \mathrm{MHz}, 298 \mathrm{~K}\right): \delta \mathrm{ppm} 0.94(\mathrm{t}, 3 \mathrm{H}, J$ $=7.0 \mathrm{~Hz}), 1.46-1.65(\mathrm{~m}, 4 \mathrm{H}), 2.38(\mathrm{t}, 2 \mathrm{H}, J=6.8 \mathrm{~Hz})$, $3.35(\mathrm{~s}, 2 \mathrm{H}), 6.58(\mathrm{~d}, 2 \mathrm{H}, J=8.4 \mathrm{~Hz}), 7.20(\mathrm{~d}, 2 \mathrm{H}, J=$ $8.4 \mathrm{~Hz})$.

${ }^{13} \mathrm{C}$ NMR $\left(\mathrm{CDCl}_{3}, 75 \mathrm{MHz}, 298 \mathrm{~K}\right): \delta \mathrm{ppm} \mathrm{13.6,} \mathrm{19.0,}$ $21.9,31.0,80.7,87.7,113.6$ (2), 114.7, 132.6 (2), 146.9.

MS (ESI+) $m / z=192.3[\mathrm{M}+\mathrm{Na}]^{+}$

Anal. Calcd for $\mathrm{C}_{12} \mathrm{H}_{15} \mathrm{~N}$ : C, 83.19; H, 8.73; N 8.08. Found C, 82.66; H, 8.44; N 7.68.

\section{4-( $p$-Tolylethynyl)benzenamine $4 d$}

Yield : $92 \%$. White solid mp $172-174^{\circ} \mathrm{C}$

IR $\left(\mathrm{cm}^{-1}\right)$ 3467, 3375, 2209, 1616, 1599, 1515, 1295, 814. 
${ }^{1} \mathbf{H}$ NMR $\left(\mathrm{CDCl}_{3}, 300 \mathrm{MHz}, 298 \mathrm{~K}\right): \delta \mathrm{ppm} 2.35$ (s, 3H), $3.81(\mathrm{~s}, 2 \mathrm{H}), 6.63(\mathrm{~d}, 2 \mathrm{H}, J=8.5 \mathrm{~Hz}), 7.12(\mathrm{~d}, 2 \mathrm{H}, J=$ $8.0 \mathrm{~Hz}), 7.33(\mathrm{~d}, 2 \mathrm{H}, J=8.5 \mathrm{~Hz}), 7.38(\mathrm{~d}, 2 \mathrm{H}, J=8.0$ $\mathrm{Hz})$.

${ }^{13} \mathrm{C}$ NMR $\left(\mathrm{CDCl}_{3}, 75 \mathrm{MHz}, 298 \mathrm{~K}\right): \delta \mathrm{ppm} 21.4,87.4$, $89.3,112.8,114.7,120.8$ (2), 129.0 (2), 131.2 (2), $132.8(2), 137.7,146.4$.

MS (APCI) $m / z=208[\mathrm{M}+\mathrm{H}]^{+}$

Anal. Calcd for $\mathrm{C}_{15} \mathrm{H}_{13} \mathrm{~N}$ : C, 86.92; H, 6.32; N, 6.76. Found C, 86.68; H, 6.33; N, 6.66 .

1-(2-(2-Methoxyphenyl)ethynyl)naphthalene 6c

Yield : 79\%; yellow oil.

IR $\left(\mathrm{cm}^{-1}\right)$ 3057, 2834, 1574, 1491, 1461, 1433, 1397, 1275, 1242, 1180, 1161, 1113, 1047, 1022, 934, 799, 772.

${ }^{1} \mathbf{H}$ NMR $\left(\mathrm{CDCl}_{3}, 300 \mathrm{MHz}, 298 \mathrm{~K}\right): \delta \mathrm{ppm} 3.82(\mathrm{~s}, 3 \mathrm{H})$, 6.80 (s, 2H), 7.18 (ddd, $1 \mathrm{H}, J=1.7 \mathrm{~Hz}, \mathrm{~J}=7.5 \mathrm{~Hz}, J=8.3$ $\mathrm{Hz}), 7.38$ (m, 4H), 7.67 (m, 3H), $8.41(\mathrm{dd}, 1 \mathrm{H}, J=1.7 \mathrm{~Hz}, J$ $=8.3 \mathrm{~Hz})$.

${ }^{13} \mathrm{C}$ NMR $\left(\mathrm{CDCl}_{3}, 75 \mathrm{MHz}, 298 \mathrm{~K}\right): 56.0,90.9,91.8$, $110.8,112.8,120.2,120.6,121.4,125.3,126.4,126.6$, $126.8,128.3,128.6,129.9,130.1,133.3,133.4,160.2$.

MS (APCI) $m / z=259[\mathrm{M}+\mathrm{H}]^{+}$

Anal. Calcd for $\mathrm{C}_{19} \mathrm{H}_{14} \mathrm{O}$ : C, 88.34; H, 5.46. Found C, 88.04; H, 5.28.

\section{tert-Butyl(2-((2-methoxyphenyl)ethynyl)phenoxy)dimethyl silane 6d}

Yield : $45 \%$; brown oil.

IR $\left(\mathrm{cm}^{-1}\right)$ 2930, 2857, 1593, 1574, 1497, 1482, 1461, 1444, 1285, 1249, 1181, 1161, 1113, 1096, 1025, 916, $838,806,780$.

${ }^{1} \mathrm{H}$ NMR $\left(\mathrm{CDCl}_{3}, 300 \mathrm{MHz}, 298 \mathrm{~K}\right): \delta \mathrm{ppm} 0.00(\mathrm{~s}$, $6 \mathrm{H}), 0.77(\mathrm{~s}, 9 \mathrm{H}), 3.63(\mathrm{~s}, 3 \mathrm{H}), 6.60(\mathrm{~m}, 4 \mathrm{H}), 6.98(\mathrm{~m}$, $2 \mathrm{H}), 7.23(\mathrm{dt}, 2 \mathrm{H}, J=1.7 \mathrm{~Hz}, J=7.7 \mathrm{~Hz}$ ).

${ }^{13} \mathrm{C}$ NMR $\left(\mathrm{CDCl}_{3}, 75 \mathrm{MHz}, 298 \mathrm{~K}\right)$ : -4.1 (2), 18.4, 25.8 (3), 55.7, 89.2, 90.9, 110.7, 113.1, 116.3, 119.8, 120.4 $121.1,129.3,129.5,133.5,133.8,156.3,160.0$.

MS (APCI) $m / z=339[\mathbf{M}+\mathrm{H}]^{+}$

Anal. Calcd for $\mathrm{C}_{21} \mathrm{H}_{26} \mathrm{O}_{2} \mathrm{Si}$ : C, 74.51; H, 7.74. Found C, 74.16; H, 7.48 .

\section{2,4-Dimethoxy-1-((2-methoxyphenyl)ethynyl)benzene 6e} Yield : $69 \%$; brown solid; $\mathrm{mp} 98^{\circ} \mathrm{C}$

IR $\left(\mathrm{cm}^{-1}\right) 2934,1606,1508,1457,1299,1243,1208,1161$, 1024,751 .

${ }^{1} \mathbf{H}$ NMR $\left(\mathrm{CDCl}_{3}, 300 \mathrm{MHz}, 298 \mathrm{~K}\right)$ : $\delta \mathrm{ppm} 3.82(\mathrm{~s}, 3 \mathrm{H})$, $3.90(\mathrm{~s}, 3 \mathrm{H}), 3.92(\mathrm{~s}, 3 \mathrm{H}), 6.45-6.50(\mathrm{~m}, 2 \mathrm{H}), 6.86-6.96(\mathrm{~m}$, $2 \mathrm{H}), 7.22-7.31(\mathrm{~m}, 1 \mathrm{H}), 7.45(\mathrm{dt}, 1 \mathrm{H}, J=8.7 \mathrm{~Hz}, J=1.2$ $\mathrm{Hz}), 7.50$ (dd, $1 \mathrm{H}, J=7.5 \mathrm{~Hz}, J=1.6 \mathrm{~Hz})$.

${ }^{13} \mathrm{C}$ NMR $\left(\mathrm{CDCl}_{3}, 75 \mathrm{MHz}, 298 \mathrm{~K}\right): \delta \mathrm{ppm} 55.5,56.1$, $88.5,90.0,98.6,104.9,105.6,110.8,113.3,120.5,129.3$, 133.5, 134.4, 159.9, 161.2, 161.3.

MS (APCI) $m / z=269[\mathrm{M}+\mathrm{H}]^{+}$

Anal. Calcd for $\mathrm{C}_{17} \mathrm{H}_{16} \mathrm{O}_{3}$ : C, 76.10; H, 6.01. Found $\mathrm{C}$, 75.87; H, 5.88.
Methyl(2-(2-(naphthalen-1-yl)ethynyl)phenyl)sulfane 6g Yield : $30 \%$; colorless oil.

${ }^{1} \mathbf{H}$ NMR $\left(\mathrm{CDCl}_{3}, 300 \mathrm{MHz}, 298 \mathrm{~K}\right): \delta 2.71(\mathrm{~s}, 3 \mathrm{H}), 7.33$ $(\mathrm{m}, 2 \mathrm{H}), 7.49(\mathrm{dt}, 1 \mathrm{H}, J=1.2 \mathrm{~Hz}, J=7.9 \mathrm{~Hz}), 7.69(\mathrm{~m}$, 4H), $7.99(\mathrm{~m}, 3 \mathrm{H}), 8.77(\mathrm{~d}, 1 \mathrm{H}, J=8.3 \mathrm{~Hz})$.

MS (APCI) $m / z=275[\mathrm{M}+\mathrm{H}]^{+}$

Anal. Calcd for $\mathrm{C}_{19} \mathrm{H}_{14} \mathrm{~S}$ : C, 83.17; H, 5.14. Found C, 82.82; H, 4.95.

\section{tert-Butyldimethyl(2-(2-(2-(methylthio)phenyl)ethynyl) phenoxy)silane $6 \mathrm{~h}$}

Yield : $52 \%$; yellow oil.

IR $\left(\mathrm{cm}^{-1}\right)$ 2361, 2218, 1994, 1574, 1497, 1482, 916, 838, 806, $780,744$.

${ }^{1} \mathbf{H}$ NMR $\left(\mathrm{CDCl}_{3}, 300 \mathrm{MHz}, 298 \mathrm{~K}\right): \delta 0.06(\mathrm{~s}, 6 \mathrm{H}), 0.83$ (s, 9H), 2.29 (s, 3H), $6.64(\mathrm{dd}, 1 \mathrm{H}, J=1.0 \mathrm{~Hz}, J=8.2 \mathrm{~Hz})$, $6.73(\mathrm{dt}, 1 \mathrm{H}, J=1.0 \mathrm{~Hz}, J=7.5 \mathrm{~Hz}), 6.89(\mathrm{dt}, 1 \mathrm{H}, J=1.2$ $\mathrm{Hz}, \mathrm{J}=7.4 \mathrm{~Hz}), 7.06(\mathrm{~m}, 3 \mathrm{H}), 7.24(\mathrm{dd}, 1 \mathrm{H}, J=1.4 \mathrm{~Hz}, J=$ $7.6 \mathrm{~Hz}), 7.34(\mathrm{dd}, 1 \mathrm{H}, J=1.8 \mathrm{~Hz}, J=7.7 \mathrm{~Hz})$.

${ }^{13} \mathrm{C}$ NMR $\left(\mathrm{CDCl}_{3}, 75 \mathrm{MHz}, 298 \mathrm{~K}\right):-4.0$ (2), 15.2, 18.4 (3), 25.9, 88.5, 90.6, 112.9, 119.9, 121.3, 124.2, 128.5, $129.7,132.0,133.9,134.5$

MS (APCI) $m / z=355[\mathrm{M}+\mathrm{H}]^{+}$

Anal. Calcd for $\mathrm{C}_{21} \mathrm{H}_{26} \mathrm{OSSi}$ : C, 71.13; H, 7.39. Found C, 70.89; H, 7.10.

(E)-1-Methoxy-2-(oct-3-en-1-ynyl)benzene 8b

Yield : $82 \%$; yellow oil

IR $\left(\mathrm{cm}^{-1}\right) 1673,1603,1510$.

${ }^{1} \mathbf{H}$ NMR $\left(\mathrm{CDCl}_{3}, 300 \mathrm{MHz}, 298 \mathrm{~K}\right): \delta \mathrm{ppm} 0.95(\mathrm{t}, 3 \mathrm{H}, J$ $=6.9 \mathrm{~Hz}), 1.34-1.44(\mathrm{~m}, 4 \mathrm{H}), 2.16(\mathrm{q}, 2 \mathrm{H}, J=6.8 \mathrm{~Hz}), 3.89$ $(\mathrm{s}, 3 \mathrm{H}), 5.75(\mathrm{~d}, 1 \mathrm{H}, J=15.5 \mathrm{~Hz}), 6.21-6.31(\mathrm{~m}, 1 \mathrm{H}), 6.85-$ $6.92(\mathrm{~m}, 2 \mathrm{H}), 7.23-7.29(\mathrm{~m}, 1 \mathrm{H}), 7.39(\mathrm{~d}, 1 \mathrm{H}, J=7.5 \mathrm{~Hz})$.

${ }^{13} \mathrm{C}$ NMR $\left(\mathrm{CDCl}_{3}, 75 \mathrm{MHz}, 298 \mathrm{~K}\right)$ : $\delta$ ppm 14.0, 22.3, $31.0,33.0,55.9,84.1,92.6,109.9,110.6,112.9$, 120.5, $129.4,133.5,145.1,159.8$

MS (ESI) $m / z=237.3[\mathrm{M}+\mathrm{Na}]^{+}$

Anal. Calcd for $\mathrm{C}_{15} \mathrm{H}_{18} \mathrm{O}$ : C, 84.07; H, 8.47. Found C, 83.93; H, 8.33.

\section{(E)-1-Methoxy-2-(4-phenylbut-3-en-1-ynyl)benzene 8c}

Yield : $91 \%$; yellow oil

IR $\left(\mathrm{cm}^{-1}\right) 3029,1592,1489,1462,1433,1274,1258,1239$, 1119, 1024, 951, 745, 690 .

${ }^{1} \mathbf{H}$ NMR $\left(\mathrm{CDCl}_{3}, 300 \mathrm{MHz}, 298 \mathrm{~K}\right): \delta \mathrm{ppm} 3.92(\mathrm{~s}, 3 \mathrm{H})$, $6.47(\mathrm{~d}, 1 \mathrm{H}, J=16.2 \mathrm{~Hz}), 6.90(\mathrm{~d}, 1 \mathrm{H}, J=8.3 \mathrm{~Hz}), 6.94(\mathrm{td}$, $1 \mathrm{H}, J=7.5 \mathrm{~Hz}, J=0.8 \mathrm{~Hz}), 7.07(\mathrm{~d}, 1 \mathrm{H}, J=16.2 \mathrm{~Hz})$, 7.27-7.38 (m, 4H), 7.42-7.48 (m, 3H).

${ }^{13} \mathrm{C}$ NMR $\left(\mathrm{CDCl}_{3}, 75 \mathrm{MHz}, 298 \mathrm{~K}\right): \delta \mathrm{ppm} 56.0,88.2$, 93.1, 108.6, 110.8, 112.8, 120.7, 126.4, 128.6, 128.8, 129.8, 133.6, 136.6, 141.1, 160.0.

MS (APCI) $m / z=235[\mathrm{M}+\mathrm{H}]^{+}$

Anal. Calcd for $\mathrm{C}_{17} \mathrm{H}_{14} \mathrm{O}$ : C, 87.15; H, 6.02. Found C, 87.01; H, 5.97.

(E)- 4-(2-Methoxyphenyl)-1-(4-methoxyphenyl)but-1-en-3-yne 8d

Yield : 59\%; yellow solid; $\mathrm{mp} 69^{\circ} \mathrm{C}$ 
IR $\left(\mathrm{cm}^{-1}\right)$ 2935, 1604, 1510, 1491, 1247, 1028, 752.

${ }^{1} \mathbf{H}$ NMR $\left(\mathrm{CDCl}_{3}, 300 \mathrm{MHz}, 298 \mathrm{~K}\right): \delta \mathrm{ppm} 3.82(\mathrm{~s}, 3 \mathrm{H})$, 3.91 (s, 3H), 6.31 (d, $1 \mathrm{H}, J=16.2 \mathrm{~Hz}), 6.86-6.95(\mathrm{~m}, 4 \mathrm{H})$, $7.02(\mathrm{~d}, 1 \mathrm{H}, J=16.2 \mathrm{~Hz}), 7.26-7.31(\mathrm{~m}, 1 \mathrm{H}), 7.37(\mathrm{~d}, 2 \mathrm{H}, J$ $=8.7 \mathrm{~Hz}), 7.44(\mathrm{dd}, 1 \mathrm{H}, J=7.5 \mathrm{~Hz}, J=1.6 \mathrm{~Hz})$.

${ }^{13} \mathbf{C}$ NMR $\left(\mathrm{CDCl}_{3}, 75 \mathrm{MHz}, 298 \mathrm{~K}\right): \delta \mathrm{ppm} 55.5,56.0$, 87.4, 93.5, 106.2, 110.7, 112.9, 114.3 (2), 120.6, 127.8 (2), $129.5,129.6,133.6,140.7,159.9,160.1$.

MS (APCI) $m / z=265(\mathrm{M}+\mathrm{H})^{+}$

Anal. Calcd for $\mathrm{C}_{18} \mathrm{H}_{16} \mathrm{O}_{2}$ : C, 81.79; H, 6.10. Found $\mathrm{C}$, 81.55; H, 5.91.

(E)-Methyl(2-(4-phenylbut-3-en-1-ynyl)phenyl)sulfane 8e Yield : 82\%; yellow oil

IR $\left(\mathrm{cm}^{-1}\right)$ 3026, 1463, 1434, 952, 746, 690.

${ }^{1} \mathbf{H}$ NMR $\left(\mathrm{CDCl}_{3}, 300 \mathrm{MHz}, 298 \mathrm{~K}\right)$ : $\delta \mathrm{ppm} 2.52(\mathrm{~s}, 3 \mathrm{H})$, $6.47(\mathrm{~d}, 1 \mathrm{H}, J=16.2 \mathrm{~Hz}), 7.08-7.19(\mathrm{~m}, 3 \mathrm{H}), 7.27-7.38(\mathrm{~m}$, 4H), 7.43-7.46 (m, 3H).

${ }^{13} \mathrm{C}$ NMR $\left(\mathrm{CDCl}_{3}, 75 \mathrm{MHz}, 298 \mathrm{~K}\right): \delta \mathrm{ppm} 15.3,89.3$, $95.5,108.1,116.9,121.7,124.3,124.4,126.5$ (2), 128.8 (2), 128.9, 132.4, 136.5, 141.6.

MS (APCI) $m / z=251(\mathrm{M}+\mathrm{H})^{+}$

Anal. Calcd for $\mathrm{C}_{17} \mathrm{H}_{14} \mathrm{~S}$ : $\mathrm{C}, 81.56 ; \mathrm{H}$, 5.64. Found $\mathrm{C}$, 81.39; H, 5.51.

(2-(4-(2-Methoxyphenyl)buta-1,3-diynyl)phenyl)(methyl) sulfane $9 \mathrm{~b}$

Yield : $70 \%$; light brown solid; $\mathrm{mp} 82-83^{\circ} \mathrm{C}$

IR $\left(\mathrm{cm}^{-1}\right)$ 2938, 1594, 1492, 1464, 1433, 1248, 1022, 703.

${ }^{1} \mathbf{H}$ NMR $\left(\mathrm{CDCl}_{3}, 300 \mathrm{MHz}, 298 \mathrm{~K}\right): \delta \mathrm{ppm} 2.51(\mathrm{~s}, 3 \mathrm{H})$, $3.91(\mathrm{~s}, 3 \mathrm{H}), 6.89$ (d, 1H, $J=8.7 \mathrm{~Hz}), 6.92(\mathrm{t}, 1 \mathrm{H}, J=7.5$ $\mathrm{Hz}), 7.09$ (t, 1H, $J=7.5 \mathrm{~Hz}), 7.17(\mathrm{~d}, 1 \mathrm{H}, J=7.9 \mathrm{~Hz}), 7.29-$ $7.36(\mathrm{~m}, 2 \mathrm{H}), 7.48-7.51(\mathrm{~m}, 2 \mathrm{H})$.

IR $\left(\mathrm{cm}^{-1}\right)$ 15.4, 56.0, 77.7, 79.5, 80.1, 80.4, 110.8, 111.2, $120.5,120.7,124.5,124.6,129.5,130.9,133.7,134.6$, 143.2, 161.5 .

MS (APCI) $m / z=279[\mathrm{M}+\mathrm{H}]^{+}$

Anal. Calcd for $\mathrm{C}_{18} \mathrm{H}_{14} \mathrm{OS}$ : C, 77.66; H, 5.07. Found C, 77.66; H, 5.01.

1-(2-Methoxyphenyl)-4-(4-methoxyphenyl)buta-1,3-diyne 9c Yield : $64 \%$; colorless solid; $\mathrm{mp} 109-110^{\circ} \mathrm{C}$

IR $\left(\mathrm{cm}^{-1}\right)$ 2936, 2837, 1601, 1508, 1489, 1247, 1171, 1024, 750 .

${ }^{1} \mathbf{H}$ NMR $\left(\mathrm{CDCl}_{3}, 300 \mathrm{MHz}, 298 \mathrm{~K}\right): \delta \mathrm{ppm} 3.82(\mathrm{~s}, 3 \mathrm{H})$, 3.90 (s, 3H), 6.84-6.94 (m, 4H), 7.32 (t, 1H, $J=7.6 \mathrm{~Hz})$, 7.45-7.48 (m, 3H).

${ }^{13} \mathrm{C}$ NMR $\left(\mathrm{CDCl}_{3}, 75 \mathrm{MHz}, 298 \mathrm{~K}\right): \delta \mathrm{ppm} 55.4,55.9$, 73.2, 77.7, 78.0, 82.5, 110.8, 111.3, 114.0, 114.2 (2), 120.6, 130.6, 134.1 (2), 134.4, 160.4, 161.5.

MS (APCI) $m / z=263[\mathrm{M}+\mathrm{H}]^{+}$

Anal. Calcd for $\mathrm{C}_{18} \mathrm{H}_{14} \mathrm{O}_{2}$ : C, 82.42; H, 5.38 Found C, $82.11 ; \mathrm{H}, 5.20$.

1-(4-(2-Methoxyphenyl)buta-1,3-diynyl)naphthalene 9d Yield : $75 \%$; beige solid; $\mathrm{mp} 82-83^{\circ} \mathrm{C}$ IR $\left(\mathrm{cm}^{-1}\right) 3059,1491,1279,1253,748$.
${ }^{1} \mathbf{H}$ NMR $\left(\mathrm{CDCl}_{3}, 300 \mathrm{MHz}, 298 \mathrm{~K}\right): \delta$ ppm $3.93(\mathrm{~s}, 3 \mathrm{H})$, 6.89-6.96 (m, 2H), 7.32-7.38 (m, 1H), 7.49-7.55 (m, 4H), 7.78-7.83 (m, 3H), $8.06(\mathrm{~s}, 1 \mathrm{H})$.

${ }^{13} \mathrm{C}$ NMR $\left(\mathrm{CDCl}_{3}, 75 \mathrm{MHz}, 298 \mathrm{~K}\right): \delta \mathrm{ppm} 56.0,74.7$, 77.9, 78.5, 82.7, 110.8, 111.2, 119.4, 120.7, 126.9, 127.3, $127.9,128.0,128.3,128.6,130.9,133.0,133.3,134.6$, 161.6.

MS (APCI) $m / z=283(\mathrm{M}+\mathrm{H})^{+}$

Anal. Calcd for $\mathrm{C}_{21} \mathrm{H}_{14} \mathrm{O}$ : C, 89.34; H, 5.00. Found C, 89.01; H, 4.79.

Typical Procedure for the PTSA-catalyzed hydration of arylalkynes under classical heating: to a stirred solution of alkyne 1a (307 mg; $1 \mathrm{mmol})$ in EtOH (2 mL), was added PTSA monohydrate (38 mg; $0.2 \mathrm{mmol}$ ). The mixture was refluxed for $3 \mathrm{~h}$, diluted in $\mathrm{H}_{2} \mathrm{O}$ and extracted with EtOAc. The combined organic layer was washed with brine and dried over $\mathrm{Na}_{2} \mathrm{SO}_{4}$. After removal of the solvent under vacuo, the crute product was purified by column chromatography on silica gel to afford $242 \mathrm{mg}$ of $\mathbf{2 a}$.

1-(4-(Diprop-2-ynylamino)phenyl)-3-ethoxypropan-1-one 2a Yield : $90 \%$; yellow solid; $\mathrm{mp} 90^{\circ} \mathrm{C}$

IR $\left(\mathrm{cm}^{-1}\right)$ 3271, 2957, 2871, 1655, 1595, 1562.

${ }^{1} \mathbf{H}$ NMR $\left(\mathrm{CDCl}_{3}, 300 \mathrm{MHz}, 298 \mathrm{~K}\right): \delta \mathrm{ppm} 1.18(\mathrm{t}, 3 \mathrm{H}, J=$ $7.0 \mathrm{~Hz}), 2.27(\mathrm{t}, 2 \mathrm{H}, J=2.3 \mathrm{~Hz}), 3.16(\mathrm{t}, 2 \mathrm{H}, J=6.8 \mathrm{~Hz})$, $3.51(\mathrm{q}, 2 \mathrm{H}, J=7.0 \mathrm{~Hz}), 3.82(\mathrm{t}, 2 \mathrm{H}, J=6.8 \mathrm{~Hz}), 4.17(\mathrm{~d}$, $4 \mathrm{H}, J=2.3 \mathrm{~Hz}), 6.88(\mathrm{~d}, 2 \mathrm{H}, J=9.1 \mathrm{~Hz}), 7.91(\mathrm{~d}, 2 \mathrm{H}, J=$ $9.1 \mathrm{~Hz})$.

${ }^{13} \mathrm{C} \mathrm{NMR}\left(\mathrm{CDCl}_{3}, 75 \mathrm{MHz}, 298 \mathrm{~K}\right): \delta$ ppm 14.9, 38.1, 39.8 (2), 65.9, 66.2, 72.9 (2), 78.3 (2), 112.9 (2), 127.6, 129.9 (2), 150.6, 196.3.

MS (ESI) $m / z=292[\mathrm{M}+\mathrm{Na}]^{+}$

Anal. Calcd for $\mathrm{C}_{17} \mathrm{H}_{19} \mathrm{NO}_{2}$ : C, 75.81; H, 7.11; N, 5.20. Found C, 75.66; H, 7.02; N, 5.05.

3-Ethoxy-1-(4-methoxyphenyl)propan-1-one $2 b^{19 e}$

Yield : $94 \%$; yellow oil

IR $\left(\mathrm{cm}^{-1}\right)$ 2974, 1673, 1599, 1575, 1510.

${ }^{1} \mathbf{H}$ NMR $\left(\mathrm{CDCl}_{3}, 300 \mathrm{MHz}, 298 \mathrm{~K}\right): \delta \mathrm{ppm} 1.14(\mathrm{t}, 3 \mathrm{H}, J=$ $7.0 \mathrm{~Hz}), 3.14(\mathrm{t}, 2 \mathrm{H}, J=6.7 \mathrm{~Hz}), 3.47(\mathrm{~d}, 2 \mathrm{H}, J=8.9 \mathrm{~Hz})$, 3.78 (s, 3H), 3.79 (t, 2H, J = 6.8 Hz), 6.87 (d, 2H, J = 8.9 $\mathrm{Hz}), 7.89(\mathrm{~d}, 2 \mathrm{H}, J=8.9 \mathrm{~Hz})$.

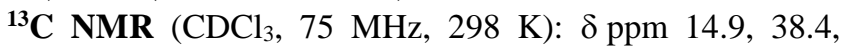
55.2, 65.8, 66.3, 113.5 (2), 130.0, 130.2 (2), 163.3, 196.7.

MS (EI) $m / z=208(18 \%)[\mathrm{M}]^{+}$

3-Methoxy-1-(4-methoxyphenyl)propan-1-one $2 \mathrm{c}^{15}$

Yield : $98 \%$; yellow oil

IR $\left(\mathrm{cm}^{-1}\right)$ 2986, 2905, 2842, 2816, 1665, 1598, 1513.

${ }^{1} \mathbf{H}$ NMR $\left(\mathrm{CDCl}_{3}, 300 \mathrm{MHz}, 298 \mathrm{~K}\right): \delta \mathrm{ppm} 3.14(\mathrm{t}, 2 \mathrm{H}, J$ $=6.6 \mathrm{~Hz}), 3.32(\mathrm{~s}, 3 \mathrm{H}), 3.76(\mathrm{t}, 2 \mathrm{H}, J=6.6 \mathrm{~Hz}), 3.81(\mathrm{~s}$, $3 \mathrm{H}), 6.88(\mathrm{~d}, 2 \mathrm{H}, J=8.9 \mathrm{~Hz}), 7.90(\mathrm{~d}, 2 \mathrm{H}, J=8.9 \mathrm{~Hz})$.

${ }^{13} \mathrm{C}$ NMR $\left(\mathrm{CDCl}_{3}, 75 \mathrm{MHz}, 298 \mathrm{~K}\right): \delta \mathrm{ppm} 38.1,55.2$, 58.6, 67.9, 113.5 (2), 130.0, 130.2 (2), 163.4, 196.6.

MS (EI) $m / z=194(23 \%)[\mathrm{M}]^{+}$ 
3-( $\left.{ }^{2} \mathrm{H}\right)$-Methoxy-1-(4-methoxyphenyl)-(2,2-2 H)propan-1-one $D-2 c$

Yield : $98 \%$; yellow oil

${ }^{1} \mathbf{H}$ NMR $\left(\mathrm{CDCl}_{3}, 300 \mathrm{MHz}, 298 \mathrm{~K}\right)$ : $\delta$ ppm 3.77 (s, 2H), $3.83(\mathrm{~s}, 3 \mathrm{H}), 6.91$ (d, 2H, $J=8.8 \mathrm{~Hz}), 7.92(\mathrm{~d}, 2 \mathrm{H}, J=8.8$ $\mathrm{Hz})$.

${ }^{13} \mathbf{C}$ NMR $\left(\mathrm{CDCl}_{3}, 75 \mathrm{MHz}, 298 \mathrm{~K}\right): \delta \mathrm{ppm} 37.5$ (q, $J=$ $19.3 \mathrm{~Hz}), 55.2,57.8(\mathrm{~m}, J=21.5 \mathrm{~Hz}), 67.8,113.6(2)$, 130.0, 130.2 (2), 163.4, 196.7.

MS (ES) $m / z=222[\mathrm{M}+\mathrm{Na}]^{+}$

3-isopropoxy-1-(4-methoxyphenyl)propan-1-one $2 d^{15}$

Yield : 66\%; pale yellow oil

${ }^{1} \mathbf{H}$ NMR $\left(\mathrm{CDCl}_{3}, 300 \mathrm{MHz}, 298 \mathrm{~K}\right): \delta \mathrm{ppm} 1.13(\mathrm{~d}, 6 \mathrm{H}, J$ $=6.1 \mathrm{~Hz}), 3.15(\mathrm{t}, 2 \mathrm{H}, J=6.8 \mathrm{~Hz}), 3.59(\mathrm{~m}, 1 \mathrm{H}), 3.81(\mathrm{~s}$, $3 \mathrm{H}), 3.82(\mathrm{t}, 2 \mathrm{H}, J=6.8 \mathrm{~Hz}), 6.89(\mathrm{~d}, 2 \mathrm{H}, J=8.9 \mathrm{~Hz}), 7.92$ $(\mathrm{d}, 2 \mathrm{H}, J=8.9 \mathrm{~Hz})$.

${ }^{13} \mathrm{C}$ NMR $\left(\mathrm{CDCl}_{3}, 75 \mathrm{MHz}, 298 \mathrm{~K}\right): \delta$ ppm 21.7 (2), 38.6, 55.0, 63.2, 71.4, 113.3 (2), 129.9, 130.0 (2), 163.1, 196.6.

MS (ESI) $m / z=245[\mathrm{M}+\mathrm{Na}]^{+}$

3-(2-Hydroxyethoxy)-1-(4-methoxyphenyl)propan-1-one $2 \mathrm{e}^{15}$ Yield : $33 \%$; colorless oil

${ }^{1} \mathbf{H}$ NMR $\left(\mathrm{CDCl}_{3}, 300 \mathrm{MHz}, 298 \mathrm{~K}\right)$ : $\delta$ ppm 2.50-2.70 (s, $1 \mathrm{H}), 3.17(\mathrm{t}, 2 \mathrm{H}, J=6.2 \mathrm{~Hz}), 3.56(\mathrm{t}, 2 \mathrm{H}, J=4.8 \mathrm{~Hz}), 3.69$ $(\mathrm{t}, 2 \mathrm{H}, J=4.8 \mathrm{~Hz}), 3.83(\mathrm{~s}, 3 \mathrm{H}), 3.88(\mathrm{t}, 2 \mathrm{H}, J=6.2 \mathrm{~Hz})$, $6.89(\mathrm{~d}, 2 \mathrm{H}, J=8.9 \mathrm{~Hz}), 7.91(\mathrm{~d}, 2 \mathrm{H}, J=8.9 \mathrm{~Hz})$.

${ }^{13} \mathrm{C}$ NMR $\left(\mathrm{CDCl}_{3}, 75 \mathrm{MHz}, 298 \mathrm{~K}\right)$ : $\delta \mathrm{ppm} \mathrm{38.1,} \mathrm{55.3,}$ 61.5, 65.9, 72.0, 113.7 (2), 129.9, 130.3 (2), 163.6, 196.9.

MS (ESI) $m / z=225[\mathrm{M}+\mathrm{Na}]^{+}$

3-hydroxy-1-(4-methoxyphenyl)propan-1-one $2 \mathrm{f}^{28}$

Yield : $75 \%$; yellow solid; $\mathrm{mp} 62^{\circ} \mathrm{C}$

${ }^{1} \mathbf{H}$ NMR $\left(\mathrm{CDCl}_{3}, 300 \mathrm{MHz}, 298 \mathrm{~K}\right): \delta \mathrm{ppm}$ 2.50-2.70 (s, $1 \mathrm{H}), 3.16(\mathrm{t}, 2 \mathrm{H}, J=5.5 \mathrm{~Hz}), 4.72(\mathrm{~s}, 3 \mathrm{H}), 4.00(\mathrm{t}, 2 \mathrm{H}, J=$ $5.5 \mathrm{~Hz}), 6.93(\mathrm{~d}, 2 \mathrm{H}, J=9.0 \mathrm{~Hz}), 7.93(\mathrm{~d}, 2 \mathrm{H}, J=9.0 \mathrm{~Hz})$.

${ }^{13} \mathrm{C}$ NMR $\left(\mathrm{CDCl}_{3}, 75 \mathrm{MHz}, 298 \mathrm{~K}\right)$ : $\delta$ ppm 39.9, 55.4, 58.1, 113.7 (2), 129.8, 130.3 (2), 163.7, 198.9.

MS (ESI) $m / z=181[\mathrm{M}+\mathrm{H}]^{+}$

4-Ethoxy-1-(4-methoxyphenyl)butan-1-one $2 \mathrm{~g}^{15}$

Yield : 85\%; yellow oil

${ }^{1} \mathbf{H}$ NMR $\left(\mathrm{CDCl}_{3}, 300 \mathrm{MHz}, 298 \mathrm{~K}\right): \delta \mathrm{ppm} 1.16(\mathrm{t}, 3 \mathrm{H}, J=$ $7.0 \mathrm{~Hz}), 1.17(\mathrm{~m}, 2 \mathrm{H}), 2.99(\mathrm{t}, 2 \mathrm{H}, J=7.2 \mathrm{~Hz}), 3.35-3.50$ $(\mathrm{m}, 4 \mathrm{H}), 3.83(\mathrm{~s}, 3 \mathrm{H}), 6.90(\mathrm{~d}, 2 \mathrm{H}, J=8.7 \mathrm{~Hz}), 7.93(\mathrm{~d}, 2 \mathrm{H}$, $J=8.7 \mathrm{~Hz})$.

${ }^{13} \mathrm{C}$ NMR $\left(\mathrm{CDCl}_{3}, 75 \mathrm{MHz}, 298 \mathrm{~K}\right): \delta \mathrm{ppm} 15.0,24.4$, 34.6, 55.2, 65.4, 69.5, 113.5 (2), 130.0, 130.1 (2), 163.3, 198.4 .

MS (ESI) $m / z=245[\mathrm{M}+\mathrm{Na}]^{+}$

6-Hydroxy-1-(4-methoxyphenyl)hexan-1-one $2 h^{15}$

Yield : $90 \%$; yellow solid; $\mathrm{mp} 60^{\circ} \mathrm{C}$

${ }^{1} \mathbf{H}$ NMR $\left(\mathrm{CDCl}_{3}, 300 \mathrm{MHz}, 298 \mathrm{~K}\right)$ : $\delta \mathrm{ppm}$ 1.40-1.60 (m, $6 \mathrm{H}), 2.92(\mathrm{t}, 2 \mathrm{H}, J=7.3 \mathrm{~Hz}), 3.65(\mathrm{t}, 2 \mathrm{H}, J=6.4 \mathrm{~Hz}), 3.85$ $(\mathrm{s}, 3 \mathrm{H}), 6.91(\mathrm{~d}, 2 \mathrm{H}, J=8.9 \mathrm{~Hz}), 7.92(\mathrm{~d}, 2 \mathrm{H}, J=8.9 \mathrm{~Hz})$.
${ }^{13} \mathrm{C}$ NMR $\left(\mathrm{CDCl}_{3}, 75 \mathrm{MHz}, 298 \mathrm{~K}\right): \delta \mathrm{ppm} 24.1,25.5$, 32.5, 38.0, 55.4, 62.6, 113.7 (2), 130.1, 130.2 (2), 163.4, 199.0.

MS (EI) $m / z=222[\mathrm{M}]^{+}$

3-Ethoxy-1-(4-methoxyphenyl)butan-1-one $2 i^{15}$

Yield : 91\%; yellow oil

IR $\left(\mathrm{cm}^{-1}\right)$ 2973, 2930, 1675, 1600, 1576, 1510.

${ }^{1} \mathbf{H}$ NMR $\left(\mathrm{CDCl}_{3}, 300 \mathrm{MHz}, 298 \mathrm{~K}\right): \delta \mathrm{ppm} 1.14(\mathrm{t}, 3 \mathrm{H}, J$ $=7.0 \mathrm{~Hz}), 1.24(\mathrm{~d}, 3 \mathrm{H}, J=6.1 \mathrm{~Hz}), 2.86(\mathrm{dd}, 1 \mathrm{H}, J=6.2$ $\mathrm{Hz}, J=15.8 \mathrm{~Hz}), 3.28(\mathrm{dd}, 1 \mathrm{H}, J=6.2 \mathrm{~Hz}, J=15.8 \mathrm{~Hz})$, 3.50 (quint, $1 \mathrm{H}, J=7.0 \mathrm{~Hz}), 3.51(\mathrm{dd}, 1 \mathrm{H}, J=7.0 \mathrm{~Hz}, J=$ $7.0 \mathrm{~Hz}), 3.86(\mathrm{~s}, 3 \mathrm{H}), 4.06(\mathrm{~m}, 1 \mathrm{H}), 6.92(\mathrm{~d}, 2 \mathrm{H}, J=8.9$ $\mathrm{Hz}), 7.94(\mathrm{~d}, 2 \mathrm{H}, J=8.9 \mathrm{~Hz})$.

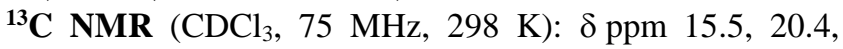
45.5, 55.4, 64.1, 72.0, 113.7 (2), 130.5, 130.6 (2), 163.5, 197.4.

MS (ESI) $m / z=223[\mathrm{M}+\mathrm{H}]^{+}$

5-Hydroxy-1-(4-methoxyphenyl)pentan-1-one $\mathbf{2} \mathbf{j}^{29}$

Yield : 45\%; colorless solid; $\mathrm{mp} 50^{\circ} \mathrm{C}$

IR $\left(\mathrm{cm}^{-1}\right)$ 3470, 1671, 1605 .

${ }^{1} \mathbf{H}$ NMR $\left(\mathrm{CDCl}_{3}, 300 \mathrm{MHz}, 298 \mathrm{~K}\right): \delta \mathrm{ppm} 1.50-2.05(\mathrm{~m}$, $5 \mathrm{H}), 3.01(\mathrm{t}, 2 \mathrm{H}, J=6.5 \mathrm{~Hz}), 3.80-3.60(\mathrm{~m}, 2 \mathrm{H}), 3.75(\mathrm{~s}$, $3 \mathrm{H}), 6.85(\mathrm{~d}, 2 \mathrm{H}, J=8.8 \mathrm{~Hz}), 7.92(\mathrm{~d}, 2 \mathrm{H}, J=8.8 \mathrm{~Hz})$.

5-Ethoxy-1-(4-methoxyphenyl)pentan-1-one $2 \mathbf{k}^{29}$

Yield : 44\%; colorless solid; $\mathrm{mp} 46^{\circ} \mathrm{C}$

IR $\left(\mathrm{cm}^{-1}\right)$ 1673, 1603, 1510.

${ }^{1} \mathbf{H}$ NMR $\left(\mathrm{CDCl}_{3}, 300 \mathrm{MHz}, 298 \mathrm{~K}\right): \delta \mathrm{ppm} 1.20(\mathrm{t}, 3 \mathrm{H}, J$ $=7.1 \mathrm{~Hz}), 1.50-2.01(\mathrm{~m}, 4 \mathrm{H}), 2.95(\mathrm{t}, 2 \mathrm{H}, J=6.5 \mathrm{~Hz}), 3.45$ $(\mathrm{t}, 2 \mathrm{H}, J=6.5 \mathrm{~Hz}), 3.50(\mathrm{q}, 2 \mathrm{H}, J=7.1 \mathrm{~Hz}), 3.86(\mathrm{~s}, 3 \mathrm{H})$, $6.92(\mathrm{~d}, 2 \mathrm{H}, J=8.9 \mathrm{~Hz}), 7.91(\mathrm{~d}, 2 \mathrm{H}, J=8.9 \mathrm{~Hz})$.

1-(4-Methoxyphenyl)hexan-1-one $2 \mathrm{I}^{30}$

Yield : $81 \%$; yellow solid; $\mathrm{mp} 60^{\circ} \mathrm{C}$

IR $\left(\mathrm{cm}^{-1}\right)$ 3077, 3011, 2956, 2931, 1676, 1601, 1254.

${ }^{1} \mathbf{H}$ NMR $\left(\mathrm{CDCl}_{3}, 300 \mathrm{MHz}, 298 \mathrm{~K}\right): \delta \mathrm{ppm} 0.90(\mathrm{t}, 3 \mathrm{H}, J=$ $5.6 \mathrm{~Hz}$ ), $1.36(\mathrm{~m}, 4 \mathrm{H}), 1.70$ (quint, $2 \mathrm{H}, J=7.3 \mathrm{~Hz}$ ), 2.89 (t, $2 \mathrm{H}, J=7.3 \mathrm{~Hz}), 3.85(\mathrm{~s}, 3 \mathrm{H}), 6.91(\mathrm{~d}, 2 \mathrm{H}, J=8.8 \mathrm{~Hz}), 7.93$ $(\mathrm{d}, 2 \mathrm{H}, J=8.8 \mathrm{~Hz})$.

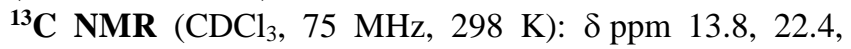
24.2, 31.5, 38.1, 55.3, 113.5 (2), 130.2 (3), 163.2, 199.0.

MS (EI) $m / z=206[\mathrm{M}]^{+}$

1-(4-Aminophenyl)-6-hydroxyhexan-1-one $\mathbf{2} \mathbf{m}^{15}$

Yield : $72 \%$; yellow solid; $\mathrm{mp} 114^{\circ} \mathrm{C}$

${ }^{1} \mathbf{H}$ NMR $\left(\mathrm{CD}_{3} \mathrm{OD}, 300 \mathrm{MHz}, 298 \mathrm{~K}\right): \delta$ ppm 1.20-1.80 (m, $6 \mathrm{H}), 2.87(\mathrm{t}, 2 \mathrm{H}, J=7.6 \mathrm{~Hz}), 3.55(\mathrm{t}, 2 \mathrm{H}, J=6.4 \mathrm{~Hz}), 6.63$ $(\mathrm{d}, 2 \mathrm{H}, J=8.8 \mathrm{~Hz}), 7.75(\mathrm{~d}, 2 \mathrm{H}, J=8.8 \mathrm{~Hz})$.

${ }^{13} \mathrm{C}$ NMR $\left(\mathrm{CD}_{3} \mathrm{OD}, 75 \mathrm{MHz}, 298 \mathrm{~K}\right): \delta \mathrm{ppm} 26.1,26.7$, 33.5, 38.6, 62.3, 114.3 (2), 126.8, 132.1(2), 153.6, 201.5.

MS (ESI) $m / z=230[\mathrm{M}+\mathrm{Na}]^{+}$

3-Hydroxy-1-(4-hydroxyphenyl)propan-1-one $2 \mathbf{n}^{30}$

Yield : $79 \%$; white solid; $\mathrm{mp} 140^{\circ} \mathrm{C}$

IR $\left(\mathrm{cm}^{-1}\right)$ 3462, 3114, 1643, 1596 
${ }^{1}$ H NMR $\left(\mathrm{CD}_{3} \mathrm{OD}, 300 \mathrm{MHz}, 298 \mathrm{~K}\right): \delta$ ppm $3.14(\mathrm{t}, 2 \mathrm{H}, J$ $=6.2 \mathrm{~Hz}), 3.93(\mathrm{t}, 2 \mathrm{H}, J=6.2 \mathrm{~Hz}), 6.84(\mathrm{~d}, 2 \mathrm{H}, J=8.9 \mathrm{~Hz})$, 7.89 (d, 2H, $J=8.9 \mathrm{~Hz})$.

${ }^{13} \mathrm{C}$ NMR $\left(\mathrm{CD}_{3} \mathrm{OD}, 75 \mathrm{MHz}, 298 \mathrm{~K}\right): \delta \mathrm{ppm} 41.7,58.9$, 116.2, 130.2, 131.8, 163.8, 199.7 .

MS (ESI) $m / z=167[\mathrm{M}+\mathrm{H}]^{+}$

3-Hydroxy-1-(2-hydroxyphenyl)propan-1-one 2o

Yield : $73 \%$.

${ }^{1} \mathbf{H}$ NMR $\left(\mathrm{CD}_{3} \mathrm{COCD}_{3}, 300 \mathrm{MHz}, 298 \mathrm{~K}\right): \delta$ ppm 2.80 (bs, $1 \mathrm{H}), 3.31(\mathrm{t}, 2 \mathrm{H}, J=8.8 \mathrm{~Hz}), 4.01(\mathrm{~m}, 2 \mathrm{H}), 6.87-7.01(\mathrm{~m}$, $2 \mathrm{H}), 7.55(\mathrm{td}, 1 \mathrm{H}, J=7.9 \mathrm{~Hz}, J=1.3 \mathrm{~Hz}), 7.98(\mathrm{dd}, 1 \mathrm{H}, J=$ $7.9 \mathrm{~Hz}, J=1.3 \mathrm{~Hz}), 12.40$ (bs, $1 \mathrm{H})$.

${ }^{13} \mathrm{C}$ NMR $\left(\mathrm{CD}_{3} \mathrm{COCD}_{3}, 75 \mathrm{MHz}, 298 \mathrm{~K}\right)$ : $\delta \mathrm{ppm} 41.0$, 57.3, 117.7, 118.8, 120.1, 130.7, 136.2, 205.1.

Anal. Calcd for $\mathrm{C}_{9} \mathrm{H}_{10} \mathrm{O}_{3}$ : C, 65.05; H, 6.07. Found $\mathrm{C}$, 64.87; H, 5.90.

\section{3-Ethoxy-1-(2-methoxyphenyl)propan-1-one 2p \\ Yield : $73 \%$; yellow oil \\ IR $\left(\mathrm{cm}^{-1}\right)$ 2974, 2868, 1672, 1597.}

${ }^{1} \mathbf{H}$ NMR $\left(\mathrm{CDCl}_{3}, 300 \mathrm{MHz}, 298 \mathrm{~K}\right): \delta \mathrm{ppm} 1.13(\mathrm{t}, 3 \mathrm{H}, J=$ $7.0 \mathrm{~Hz}), 3.23(\mathrm{t}, 2 \mathrm{H}, J=6.8 \mathrm{~Hz}), 3.45(\mathrm{q}, 2 \mathrm{H}, J=7.0 \mathrm{~Hz})$, $3.76(\mathrm{t}, 2 \mathrm{H}, J=6.7 \mathrm{~Hz}), 3.83(\mathrm{~s}, 3 \mathrm{H}), 6.85-7.00(\mathrm{~m}, 2 \mathrm{H})$, $7.35-7.45(\mathrm{~m}, 1 \mathrm{H}), 7.65$ (dd, $1 \mathrm{H}, J=7.8 \mathrm{~Hz}, J=1.8 \mathrm{~Hz})$.

${ }^{13} \mathbf{C}$ NMR $\left(\mathrm{CDCl}_{3}, 75 \mathrm{MHz}, 298 \mathrm{~K}\right): \delta \mathrm{ppm} 14.9,43.9$, 55.2 , 65.6, 66.0, 111.3, 120.3, 127.9, 130.0, 133.2, 158.4, 200.0 .

MS (EI) $m / z=208[\mathrm{M}]^{+}$

Anal. Calcd for $\mathrm{C}_{12} \mathrm{H}_{16} \mathrm{O}_{3}$ : C, 69.21; H, 7.74. Found C, 69.00; H, 7.52.

\section{1-(2-Methoxyphenyl)hexan-1-one $2 q^{31}$}

Yield : $80 \%$; colorless oil

IR $\left(\mathrm{cm}^{-1}\right)$ 2928, 2858, 1674, 1597.

${ }^{1} \mathbf{H}$ NMR $\left(\mathrm{CDCl}_{3}, 300 \mathrm{MHz}, 298 \mathrm{~K}\right): \delta \mathrm{ppm} 0.87(\mathrm{t}, 3 \mathrm{H}, J=$ $6.9 \mathrm{~Hz}), 1.25-1.35(\mathrm{~m}, 4 \mathrm{H}), 1.55-1.75(\mathrm{~m}, 2 \mathrm{H}), 2.92(\mathrm{t}, 2 \mathrm{H}$, $J=7.5 \mathrm{~Hz}), 3.84(\mathrm{~s}, 3 \mathrm{H}), 6.85-7.00(\mathrm{~m}, 2 \mathrm{H}), 7.30-7.45(\mathrm{~m}$, $1 \mathrm{H}), 7.61(\mathrm{dd}, 1 \mathrm{H}, J=7.6 \mathrm{~Hz}, J=1.8 \mathrm{~Hz})$.

${ }^{13} \mathrm{C}$ NMR $\left(\mathrm{CDCl}_{3}, 75 \mathrm{MHz}, 298 \mathrm{~K}\right): \delta \mathrm{ppm} 13.7,22.3$, 23.9, 31.4, 43.5, 55.2, 111.4, 120.4, 128.7, 129.9, 132.8, 158.1, 202.9

MS (EI) $m / z=206[\mathrm{M}]^{+}$

3-Ethoxy-1-(4-(3-phenylprop-2-ynyloxy)phenyl)propan-1one $2 \mathbf{t}$

Yield : 76\%; yellow solid; $\mathrm{mp} 61^{\circ} \mathrm{C}$

IR $\left(\mathrm{cm}^{-1}\right)$ 2939, 2803, 1669, 1595.

${ }^{1} \mathbf{H}$ NMR $\left(\mathrm{CDCl}_{3}, 300 \mathrm{MHz}, 298 \mathrm{~K}\right): \delta \mathrm{ppm} 1.18(\mathrm{t}, 3 \mathrm{H}, J=$ $7.0 \mathrm{~Hz}), 3.20(\mathrm{t}, 2 \mathrm{H}, J=6.7 \mathrm{~Hz}), 3.52(\mathrm{q}, 2 \mathrm{H}, J=7.0 \mathrm{~Hz})$, $3.84(\mathrm{t}, 2 \mathrm{H}, J=6.7 \mathrm{~Hz}), 4.96(\mathrm{~s}, 2 \mathrm{H}), 7.06(\mathrm{~d}, 2 \mathrm{H}, J=9.0$ $\mathrm{Hz}), 7.30-7.40$ (m, 3H), 7.40-7.50 (m, 2H), 7.97 (d, 2H, $J=$ $9.0 \mathrm{~Hz})$.

${ }^{13} \mathrm{C}$ NMR $\left(\mathrm{CDCl}_{3}, 75 \mathrm{MHz}, 298 \mathrm{~K}\right): \delta \mathrm{ppm} \mathrm{15.0,} \mathrm{38.6,}$ 56.6, 65.8, 66.4, 83.0, 87.7, 114.6 (2), 121.9, 128.2 (2), $128.8,130.3$ (2), 130.7, 131.7 (2), 161.5, 196.9.

MS (ESI) $m / z=309[\mathrm{M}+\mathrm{H}]^{+}$
Anal. Calcd for $\mathrm{C}_{20} \mathrm{H}_{20} \mathrm{O}_{3}$ : C, 77.90; H, 6.54. Found C, 77.54; H, 6.23.

Typical procedure for the synthesis of ketones under microwaves irradiation.

To an Emrys Optimizer $0.5-2 \mathrm{~mL}$ pyrex reaction vessel were added alkyne $(1 \mathrm{mmol})$ and PTSA (see tables for quantity) in EtOH $(1 \mathrm{~mL})$. The reaction vessel was then placed in the Emrys Optimizer and exposed to microwaves irradiation according to the following specifications: temperature, time (see tables), fixed hold time: on, sample absorption: high, pre-stirring: 60s. After cooling to room temperature, $\mathrm{H}_{2} \mathrm{O}(3 \mathrm{~mL})$ was added to the crude and the mixture was extracted with EtOAc $(3 \times 2 \mathrm{~mL})$. Organic layers were dried, concentrated and the crude mixture was purified by column chromatography on silica gel.

\section{1-(3-Methoxyphenyl)-hexan-1-one 2r}

Yield : $60 \%$.

IR $\left(\mathrm{cm}^{-1}\right)$ 2954, 2932, 1684, 1596, 1260.

${ }^{1} \mathbf{H}$ NMR $\left(\mathrm{CDCl}_{3}, 300 \mathrm{MHz}, 298 \mathrm{~K}\right)$ : $\delta$ ppm $0.89(\mathrm{t}, 3 \mathrm{H}, J=$ $7.6 \mathrm{~Hz}), 1,27-1.40(\mathrm{~m}, 4 \mathrm{H}), 1.70(\mathrm{~m}, 2 \mathrm{H}), 2.92(\mathrm{t}, 2 \mathrm{H}, J=$ $7.6 \mathrm{~Hz}$ ), 3.81 (s, 3H), 7.07 (ddd, $1 \mathrm{H}, J=8.1 \mathrm{~Hz}, J=2.8 \mathrm{~Hz}$, $J=0.9 \mathrm{~Hz}), 7.35(\mathrm{dd}, 1 \mathrm{H}, J=8.1 \mathrm{~Hz}, J=8.1 \mathrm{~Hz}), 7.46(\mathrm{dd}$, $1 \mathrm{H}, J=2.8 \mathrm{~Hz}, J=1.7 \mathrm{~Hz}), 7.51(\mathrm{ddd}, 1 \mathrm{H}, J=8.1 \mathrm{~Hz}, J=$ $1.7 \mathrm{~Hz}, J=0.9 \mathrm{~Hz})$.

${ }^{13} \mathrm{C}$ NMR $\left(\mathrm{CDCl}_{3}, 75 \mathrm{MHz}, 298 \mathrm{~K}\right)$ : $\delta \mathrm{ppm} 14.0,22.6$, 24.2, 31.6, 38.7, 55.4, 112.3, 119.2, 120.6, 129.4, 138.4, 159.7, 202.2.

Anal. Calcd for $\mathrm{C}_{13} \mathrm{H}_{18} \mathrm{O}_{2}$ : C, 75.69; H, 8.80. Found C, 75.35; H, 8.54.

\section{1-Phenylhexan-1-one $\mathbf{2} \mathbf{s}^{30}$}

Yield : $89 \%$; colorless oil

IR $\left(\mathrm{cm}^{-1}\right)$ 2930, 1683, 1597, 1448, 745.

${ }^{1} \mathbf{H}$ NMR $\left(\mathrm{CDCl}_{3}, 300 \mathrm{MHz}, 298 \mathrm{~K}\right): \delta$ ppm $0.91(\mathrm{t}, 3 \mathrm{H}, J=$ $7.2 \mathrm{~Hz}$ ), 1.34-1.44 (m, 4H), 1.75 (quint, $2 \mathrm{H}, J=7.2 \mathrm{~Hz}$ ), $2.96(\mathrm{t}, 2 \mathrm{H}, J=7.2 \mathrm{~Hz}), 7.40-7.59(\mathrm{~m}, 3 \mathrm{H}), 7.93-7.98(\mathrm{dt}$, $2 \mathrm{H}, J=5.8 \mathrm{~Hz}, J=1.4 \mathrm{~Hz})$.

${ }^{13} \mathrm{C}$ NMR $\left(\mathrm{CDCl}_{3}, 75 \mathrm{MHz}, 298 \mathrm{~K}\right): \delta \mathrm{ppm} 13.9,22.5$, 24.1, 31.5, 38.6, 128.0 (2), 128.5 (2), 132.8, 137.2, 200.5.

\section{4-Ethoxy-4-(4-isopropylphenyl)-1-(4-methoxyphenyl)-butan-1- one $2 \mathrm{u}$ \\ Yield : $69 \%$. \\ IR $\left(\mathrm{cm}^{-1}\right)$ 2960, 1672, 1596, 1509, 1261, 1165, 987, 828.}

${ }^{1} \mathbf{H}$ NMR $\left(\mathrm{CDCl}_{3}, 300 \mathrm{MHz}, 298 \mathrm{~K}\right): \delta \mathrm{ppm} 1.15(\mathrm{t}, 3 \mathrm{H}, J=$ $6.9 \mathrm{~Hz}), 1.24(\mathrm{~d}, 6 \mathrm{H}, J=6.6 \mathrm{~Hz}), 2.08-2.17(\mathrm{~m}, 2 \mathrm{H}), 2.90$ $(\mathrm{q}, 1 \mathrm{H}, J=6.9 \mathrm{~Hz}), 3.01(\mathrm{td}, 2 \mathrm{H}, J=7.2 \mathrm{~Hz}, J=1.8 \mathrm{~Hz})$, $3.26(\mathrm{~m}, 2 \mathrm{H}), 3.86(\mathrm{~s}, 3 \mathrm{H}), 4.31(\mathrm{t}, 1 \mathrm{H}, J=6.3 \mathrm{~Hz}), 6.92(\mathrm{~d}$, $2 \mathrm{H}, J=8.8 \mathrm{~Hz}), 7.12-7.25(\mathrm{~m}, 4 \mathrm{H}), 7.92(\mathrm{~d}, 2 \mathrm{H}, J=8.8$ $\mathrm{Hz})$.

${ }^{13} \mathrm{C}$ NMR $\left(\mathrm{CDCl}_{3}, 75 \mathrm{MHz}, 298 \mathrm{~K}\right): \delta \mathrm{ppm} 15.3,24.0(2)$, 32.9, 33.8, 34.4, 55.4, 64.1, 80.8, 113.6 (2), 126.4 (4), 130.3 (3), 139.9, 148.0, 163.3, 198.8 .

Anal. Calcd for $\mathrm{C}_{22} \mathrm{H}_{28} \mathrm{O}_{3}$ : C, 77.61; H, 8.29. Found $\mathrm{C}$, $77.62 ; \mathrm{H}, 8.36$. 
Ethyl 4-(4-methoxyphenyl)-4-oxobutanoate $\mathbf{2} \mathbf{v}^{30}$

Yield : $51 \%$.

IR $\left(\mathrm{cm}^{-1}\right)$ 2980, 2841, 1730, 1599, 1576, 1510, 1249, 1217, $1163,1023,833,799$.

${ }^{1} \mathbf{H}$ NMR $\left(\mathrm{CDCl}_{3}, 300 \mathrm{MHz}, 298 \mathrm{~K}\right): \delta \mathrm{ppm} 1.25(\mathrm{t}, 3 \mathrm{H}, J=$ $7.1 \mathrm{~Hz}), 2.72(\mathrm{t}, 2 \mathrm{H}, J=6.7 \mathrm{~Hz}), 3.25(\mathrm{t}, 2 \mathrm{H}, J=6.7 \mathrm{~Hz})$, $3.85(\mathrm{~s}, 3 \mathrm{H}), 4.10(\mathrm{q}, 2 \mathrm{H}, J=7.1 \mathrm{~Hz}), 6.90(\mathrm{~d}, 2 \mathrm{H}, J=9.0$ $\mathrm{Hz}), 7.95(\mathrm{~d}, 2 \mathrm{H}, J=9.0 \mathrm{~Hz})$.

${ }^{13} \mathbf{C}$ NMR $\left(\mathrm{CDCl}_{3}, 75 \mathrm{MHz}, 298 \mathrm{~K}\right): \delta \mathrm{ppm} 14.1,28.4$, 33.0, 55.4, 60.5, 113.7 (2), 129.7 (2), 130.2, 163.5, 173.0, 196.6.

\section{1-(4-Methoxyphenyl)-ethanone $2 \mathbf{w}^{30}$}

Yield : $77 \%$.

IR $\left(\mathrm{cm}^{-1}\right)$ 1672, 1600, 1312, 1205.

${ }^{1} \mathbf{H}$ NMR $\left(\mathrm{CDCl}_{3}, 300 \mathrm{MHz}, 298 \mathrm{~K}\right): \delta \mathrm{ppm} 2.55(\mathrm{~s}, 3 \mathrm{H})$, $3.87(\mathrm{~s}, 3 \mathrm{H}), 6.92(\mathrm{~d}, 2 \mathrm{H}, J=8.9 \mathrm{~Hz}), 7.95(\mathrm{~d}, 2 \mathrm{H}, J=8.9$ $\mathrm{Hz})$.

${ }^{13} \mathrm{C}$ NMR $\left(\mathrm{CDCl}_{3}, 75 \mathrm{MHz}, 298 \mathrm{~K}\right): \delta \mathrm{ppm} 26.7,55.9$, 114.1 (2), 130.7, 131.0 (2), 163.9, 197.2.

1-(4-Aminophenyl)-hexan-1-one $2 \mathrm{x}^{30}$

Yield : $91 \%$.

IR $\left(\mathrm{cm}^{-1}\right)$ 3462, 3355, 2933, 1627,1587, 1560, 1318, 1258 , $1210,1176,818$

${ }^{1} \mathbf{H}$ NMR $\left(\mathrm{CDCl}_{3}, 300 \mathrm{MHz}, 298 \mathrm{~K}\right): \delta \mathrm{ppm} 0.90(\mathrm{t}, 3 \mathrm{H}, J=$ $6.6 \mathrm{~Hz}), 1.36(\mathrm{~m}, 4 \mathrm{H}), 1.73(\mathrm{q}, 2 \mathrm{H}, J=7.3 \mathrm{~Hz}), 2.85(\mathrm{t}, 2 \mathrm{H}$, $J=7.3 \mathrm{~Hz}), 4.06\left(\mathrm{bs}, 2 \mathrm{H}, \mathrm{NH}_{2}\right), 6.64(\mathrm{~d}, 2 \mathrm{H}, J=8.5 \mathrm{~Hz})$, $7.81(\mathrm{~d}, 2 \mathrm{H}, J=8.5 \mathrm{~Hz})$

${ }^{13} \mathrm{C}$ NMR $\left(\mathrm{CDCl}_{3}, 75 \mathrm{MHz}, 298 \mathrm{~K}\right): \delta \mathrm{ppm} 13.8,22.4$ 24.5, 31.5, 37.8, 113.5 (2), 127.2, 130.3 (2), 151.2, 198.8.

1-(4-Methoxyphenyl)-2-p-tolyl-ethanone $5 \mathbf{a}^{19 \mathrm{i}}$

Yield : $97 \%$.

IR $\left(\mathrm{cm}^{-1}\right)$ 3005, 2901, 1678, 1596, 1501, 1321, 1254, 1177, $1027,995,829,774$

${ }^{1} \mathbf{H}$ NMR $\left(\mathrm{CDCl}_{3}, 300 \mathrm{MHz}, 298 \mathrm{~K}\right): \delta \mathrm{ppm} 2.32(\mathrm{~s}, 3 \mathrm{H})$, $3.86(\mathrm{~s}, 3 \mathrm{H}), 4.19(\mathrm{~s}, 2 \mathrm{H}), 6.92(\mathrm{~d}, 2 \mathrm{H}, J=8.7 \mathrm{~Hz}), 7.12(\mathrm{~d}$, $2 \mathrm{H}, J=8.1 \mathrm{~Hz}), 7.16(\mathrm{~d}, 2 \mathrm{H}, J=8.1 \mathrm{~Hz}), 7.98(\mathrm{~d}, 2 \mathrm{H}, J=$ $8.7 \mathrm{~Hz})$

${ }^{13} \mathrm{C}$ NMR $\left(\mathrm{CDCl}_{3}, 75 \mathrm{MHz}, 298 \mathrm{~K}\right): \delta \mathrm{ppm} 21.0,44.9$ 55.4, 113.7 (2), 129.2 (2), 129.3 (2), 129.7, 130.9 (2), $131.8,136.3,163.4,196.4$.

1-(4-Methoxyphenyl)-2-(4-bromophenyl)-ethanone $5 b^{19 \mathrm{i}}$

Yield : $98 \%$.

IR $\left(\mathrm{cm}^{-1}\right) 33017,1675,1598,1573,1257,1177$

${ }^{1} \mathbf{H}$ NMR $\left(\mathrm{CDCl}_{3}, 300 \mathrm{MHz}, 298 \mathrm{~K}\right): \delta$ ppm $3.89(\mathrm{~s}, 3 \mathrm{H})$, $4.18(\mathrm{~s}, 2 \mathrm{H}), 6.93(\mathrm{dd}, 2 \mathrm{H}, J=6.6 \mathrm{~Hz}, J=1.8 \mathrm{~Hz}), 7.14$ $(\mathrm{dd}, 2 \mathrm{H}, J=7.5 \mathrm{~Hz}, J=2.2 \mathrm{~Hz}), 7.44(\mathrm{dd}, 2 \mathrm{H}, J=6.6 \mathrm{~Hz}, J$ $=1.8 \mathrm{~Hz}), 7.97(\mathrm{dd}, 2 \mathrm{H}, J=7.5 \mathrm{~Hz}, J=2.2 \mathrm{~Hz})$.

${ }^{13} \mathrm{C}$ NMR $\left(\mathrm{CDCl}_{3}, 75 \mathrm{MHz}, 298 \mathrm{~K}\right): \delta \mathrm{ppm} 44.5,55.5$ 113.9 (2), 120.8, 128.5, 130.8 (2), 131.2 (2), 131.7 (2), $134.0,163.7,195.5$.

\section{2-Phenyl-1-p-tolyl-ethanone $5 \mathbf{c}^{191}$}

Yield : $80 \%$.

IR $\left(\mathrm{cm}^{-1}\right) 1680,1603,1334,1222,1198,1172,814,729$.
${ }^{1} \mathbf{H}$ NMR $\left(\mathrm{CDCl}_{3}, 300 \mathrm{MHz}, 298 \mathrm{~K}\right): 2.42$ (s, 3H), 4.29 (s, $2 \mathrm{H}), 7.25-7.44(\mathrm{~m}, 7 \mathrm{H}), 7.95(\mathrm{~d}, 2 \mathrm{H}, J=8.1 \mathrm{~Hz})$.

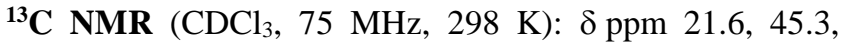
126.7, 128.6 (2), 128.7 (2), 129.2 (2), 129.4 (2), 134.0, $134.7,143.9,197.3$.

\section{1-(4-Aminophenyl)-2-p-tolyl-ethanone $5 \mathbf{d}^{19 \mathrm{i}}$}

Yield : $79 \%$.

IR $\left(\mathrm{cm}^{-1}\right) 3472,3356,1656,1630,1587,1567,1335,1316$, $1232,1174,814$.

${ }^{1} \mathbf{H}$ NMR $\left(\mathrm{CDCl}_{3}, 300 \mathrm{MHz}, 298 \mathrm{~K}\right): \delta \mathrm{ppm} 2.31$ (s, 3H), $4.14(\mathrm{~m}, 4 \mathrm{H}), 6.62(\mathrm{~d}, 2 \mathrm{H}, J=8.7 \mathrm{~Hz}), 7.11(\mathrm{~d}, 2 \mathrm{H}, J=7.8$ $\mathrm{Hz}), 7.16(\mathrm{~d}, 2 \mathrm{H}, J=7.8 \mathrm{~Hz}), 7.86(\mathrm{~d}, 2 \mathrm{H}, J=8.7 \mathrm{~Hz})$.

${ }^{13} \mathrm{C}$ NMR $\left(\mathrm{CDCl}_{3}, 75 \mathrm{MHz}, 298 \mathrm{~K}\right): \delta \mathrm{ppm} 21.0,44.6$, 113.7 (2), 127.1, 129.1 (2), 129.2 (2), 131.1 (2), 132.3, $136.1,151.1,196.0$.

\section{2-Phenyl-1-(3,4,5-trimethoxyphenyl)-ethanone 5e $\mathrm{e}^{19 \mathrm{i}}$} Yield : $68 \%$

IR $\left(\mathrm{cm}^{-1}\right)$ 2940, 2360, 1676, 1582, 1504, 1453, 1411, 1331, 1152, 1000, 697

${ }^{1} \mathbf{H}$ NMR $\left(\mathrm{CDCl}_{3}, 300 \mathrm{MHz}, 298 \mathrm{~K}\right): \delta \mathrm{ppm} 3.85(\mathrm{~s}, 6 \mathrm{H})$, $3.87(\mathrm{~s}, 3 \mathrm{H}), 4.22(\mathrm{~s}, 2 \mathrm{H}), 7.23-7.33(\mathrm{~m}, 7 \mathrm{H})$.

${ }^{13} \mathrm{C} \mathrm{NMR}\left(\mathrm{CDCl}_{3}, 75 \mathrm{MHz}, 298 \mathrm{~K}\right): \delta \mathrm{ppm} 45.6,56.2(2)$, $60.9,106.3$ (2), 126.9, 128.7 (2), 129.2 (2), 131.7, 134.8, $141.6,153.0(2), 196.4$

\section{1-(4-Methoxyphenyl)-2-(3,4,5-trimethoxyphenyl)-ethanone $\mathbf{5 f}^{19 \mathrm{e}}$}

Yield : $55 \%$.

IR $\left(\mathrm{cm}^{-1}\right)$ 2935, 2834, 1675, 1596, 1509, 1122.

${ }^{1} \mathbf{H}$ NMR $\left(\mathrm{CDCl}_{3}, 300 \mathrm{MHz}, 298 \mathrm{~K}\right): \delta \mathrm{ppm} 3.81(\mathrm{~s}, 3 \mathrm{H})$, $3.83(\mathrm{~s}, 6 \mathrm{H}), 3.86(\mathrm{~s}, 3 \mathrm{H}), 4.15(\mathrm{~s}, 2 \mathrm{H}), 6.46(\mathrm{~s}, 2 \mathrm{H}), 6.93$ $(\mathrm{d}, 2 \mathrm{H}, J=8.9 \mathrm{~Hz}), 7.98(\mathrm{~d}, 2 \mathrm{H}, J=8.9 \mathrm{~Hz})$.

${ }^{13} \mathrm{C} \mathrm{NMR}\left(\mathrm{CDCl}_{3}, 75 \mathrm{MHz}, 298 \mathrm{~K}\right): \delta$ ppm 45.4, 55.4, 56.1 (2), 60.8, 106.4 (2), 113.8 (2), 129.6, 130.5 (2), 130.9, $136.8,153.3(2), 163.6,196.1$

\section{2-(4-Methoxyphenyl)benzofuran $7 \mathbf{a}^{32}$}

Yield : $79 \%$; colorless solid; $\mathrm{mp} 148-150^{\circ} \mathrm{C}$

IR $\left(\mathrm{cm}^{-1}\right)$ 2960, 2837, 1606, 1502, 1451, 1241, 1022, 835 , 818,700 .

${ }^{1} \mathbf{H}$ NMR $\left(\mathrm{CDCl}_{3}, 300 \mathrm{MHz}, 298 \mathrm{~K}\right): \delta \mathrm{ppm} 3.87(\mathrm{~s}, 3 \mathrm{H})$, $6.89(\mathrm{~d}, 1 \mathrm{H}, J=0.9 \mathrm{~Hz}), 6.99(\mathrm{~d}, 2 \mathrm{H}, J=9.0 \mathrm{~Hz}), 7.19-7,29$ $(\mathrm{m}, 2 \mathrm{H}), 7.50-7.58(\mathrm{~m}, 2 \mathrm{H}), 7.81(\mathrm{~d}, 2 \mathrm{H}, J=9.0 \mathrm{~Hz})$.

${ }^{13} \mathbf{C}$ NMR $\left(\mathrm{CDCl}_{3}, 75 \mathrm{MHz}, 298 \mathrm{~K}\right): \delta$ ppm 55.3, 99.7, $111.0,114.2$ (2), 120.5, 122.8, 123.3, 123.7, 126.4 (2), $129.5,154.7,156.0,159.9$.

MS (APCI) $m / z=225(\mathrm{M}+\mathrm{H})^{+}$

2-(2-Methoxyphenyl)benzofuran $\mathbf{7} \mathbf{b}^{32}$

Yield : $83 \%$; colorless solid; $\mathrm{mp} 78-80^{\circ} \mathrm{C}$

IR $\left(\mathrm{cm}^{-1}\right)$ 2939, 1492, 1446, 1282, 1247, 1015, 742.

${ }^{1} \mathbf{H}$ NMR $\left(\mathrm{CDCl}_{3}, 300 \mathrm{MHz}, 298 \mathrm{~K}\right): \delta \mathrm{ppm} 3.89$ (s, 3H), $6.90(\mathrm{~d}, 1 \mathrm{H}, J=8.3 \mathrm{~Hz}), 6.98(\mathrm{td}, 1 \mathrm{H}, J=7.6 \mathrm{~Hz}, J=1.0$ $\mathrm{Hz}), 7.09-7.26(\mathrm{~m}, 4 \mathrm{H}), 7.40-7.43(\mathrm{~m}, 1 \mathrm{H}), 7.48-7.51(\mathrm{~m}$, $1 \mathrm{H}), 7.98(\mathrm{dd}, 1 \mathrm{H}, J=7.8 \mathrm{~Hz}, J=1.7 \mathrm{~Hz})$ 
${ }^{13} \mathrm{C}$ NMR $\left(\mathrm{CDCl}_{3}, 75 \mathrm{MHz}, 298 \mathrm{~K}\right): \delta \mathrm{ppm} 55.6,106.5$, $111.0,111.2,119.5,120.9,121.2,122.8,124.2,127.2$, 129.4, 129.9, 152.3, 154.0, 166.7 .

MS (APCI) $m / z=225(\mathrm{M}+\mathrm{H})^{+}$

2-(Naphthalen-1-yl)benzofuran $7 \mathbf{c}^{32}$

Yield : 93\%; yellow solid; $\mathrm{mp} 161-162^{\circ} \mathrm{C}$

IR $\left(\mathrm{cm}^{-1}\right)$ 3055, 1452, 1257, 979, 793, 771, 737.

${ }^{1} \mathbf{H}$ NMR $\left(\mathrm{CDCl}_{3}, 300 \mathrm{MHz}, 298 \mathrm{~K}\right): \delta \mathrm{ppm} 7.11(\mathrm{~s}, 1 \mathrm{H})$, 7.29-7.40 (m, 2H), 7.55-7.64 (m, 4H), $7.69(\mathrm{~d}, 1 \mathrm{H}, J=7.8$ $\mathrm{Hz}), 7.91-7.95(\mathrm{~m}, 3 \mathrm{H}), 8.50(\mathrm{~d}, 1 \mathrm{H})$.

${ }^{13} \mathrm{C}$ NMR $\left(\mathrm{CDCl}_{3}, 75 \mathrm{MHz}, 298 \mathrm{~K}\right)$ : $\delta$ ppm 106.1, 111.4, $121.1,123.1,124.5,125.4,125.7,126.3,127.0,127.5$, $128.4,128.8,129.2,129.7,130.9,134.1,155.1,155.8$.

MS (APCI) $m / z=245(\mathrm{M}+\mathrm{H})^{+}$

\section{2-(Benzofuran-2-yl)phenol 7d $\mathbf{d}^{33}$}

Yield : $61 \%$; colorless solid; $\mathrm{mp} 97^{\circ} \mathrm{C}$.

IR $\left(\mathrm{cm}^{-1}\right) 3451,3352,1590,1446,1212,1017,743$

${ }^{1} \mathbf{H}$ NMR $\left(\mathrm{CDCl}_{3}, 300 \mathrm{MHz}, 298 \mathrm{~K}\right): \delta \mathrm{ppm}$ 6.72-6.78 (m, $2 \mathrm{H}), 6.84(\mathrm{~s}, 1 \mathrm{H}), 6.91(\mathrm{~s}, 1 \mathrm{H}), 6.99-7.09(\mathrm{~m}, 3 \mathrm{H}), 7.28(\mathrm{~m}$, $1 \mathrm{H}), 7.35(\mathrm{~m}, 1 \mathrm{H}), 7.46(\mathrm{~m}, 1 \mathrm{H})$.

${ }^{13} \mathrm{C}$ NMR $\left(\mathrm{CDCl}_{3}, 75 \mathrm{MHz}, 298 \mathrm{~K}\right)$ : $\delta$ ppm 103.5, 111.2, 116.2 , 117.5, 120.9, 121.1, 123.6, 124.6, 127.3, 128.6, $130.4,153.5,154.1,154.4$.

MS (APCI+) $m / z=211[\mathrm{M}+\mathrm{H}]^{+}$

\section{2-(2,4-Dimethoxyphenyl)benzofuran 7e}

Yield : $65 \%$; colorless solid; $\mathrm{mp} 49-52^{\circ} \mathrm{C}$.

IR $\left(\mathrm{cm}^{-1}\right) 2937,2836,1610,1502,1252,1208,1158,1029$, 796, 740 .

${ }^{1} \mathbf{H}$ NMR $\left(\mathrm{CDCl}_{3}, 300 \mathrm{MHz}, 298 \mathrm{~K}\right): \delta$ ppm d $3.90(\mathrm{~s}, 3 \mathrm{H})$, $4.01(\mathrm{~s}, 3 \mathrm{H}), 6.61-6.68(\mathrm{~m}, 2 \mathrm{H}), 7.22-7.32(\mathrm{~m}, 3 \mathrm{H}), 7.54$ $(\mathrm{dt}, 1 \mathrm{H}, J=6.6 \mathrm{~Hz}, J=1.1 \mathrm{~Hz}), 7.62(\mathrm{~m}, 1 \mathrm{H}), 8.03(\mathrm{~d}, 1 \mathrm{H}$, $J=8.6 \mathrm{~Hz})$.

${ }^{13} \mathrm{C}$ NMR $\left(\mathrm{CDCl}_{3}, 75 \mathrm{MHz}, 298 \mathrm{~K}\right): \delta$ ppm $55.6(2), 98.9$, $104.4,105.0,110.7,112.9,120.8,122.7,123.7,128.1$, $130.2,152.6,153.8,157.9,161.1$

MS (APCI+) $m / z=255.0(\mathrm{M}+\mathrm{H})^{+}$

Anal. Calcd for $\mathrm{C}_{16} \mathrm{H}_{14} \mathrm{O}_{3}$ : C, 75.57; H, 5.55. Found C, 75.39; H, 5.41.

\section{2-(4-Methoxyphenyl)benzo[b]thiophene $7 \mathrm{f}^{30}$}

Yield : 94\%; colorless solid; $\mathrm{mp} 199-201^{\circ} \mathrm{C}$

IR $\left(\mathrm{cm}^{-1}\right)$ 2357, 1604, 1497, 1434, 1244, 1077, 1030, 820, 746.

${ }^{1} \mathbf{H}$ NMR $\left(\mathrm{CDCl}_{3}, 300 \mathrm{MHz}, 298 \mathrm{~K}\right): \delta \mathrm{ppm} 3.86(\mathrm{~s}, 3 \mathrm{H})$, $6.96(\mathrm{~d}, 2 \mathrm{H}, J=8.8 \mathrm{~Hz}), 7.28-7.37(\mathrm{~m}, 2 \mathrm{H}), 7.43(\mathrm{~s}, 1 \mathrm{H})$, $7.65(\mathrm{~d}, 2 \mathrm{H}, J=8.7 \mathrm{~Hz}), 7.74(\mathrm{~d}, 1 \mathrm{H}, J=7.2 \mathrm{~Hz}), 7.81$ (d, $1 \mathrm{H}, J=7.7 \mathrm{~Hz})$.

${ }^{13} \mathrm{C} \mathrm{NMR}\left(\mathrm{CDCl}_{3}, 75 \mathrm{MHz}, 298 \mathrm{~K}\right)$ : $\delta$ ppm 55.4, 114.3 (2), $118.2,122.2$, 123.2, 123.9, 124.4, 127.0, 127.7 (2), 139.2, 140.9, 144.1, 159.8.

MS (APCI) $m / z=241(\mathrm{M}+\mathrm{H})^{+}$

2-(Naphthalen-1-yl)benzo[b]thiophene $7 \mathbf{g}^{34}$

Yield : $94 \%$; beige solid; mp $106-108^{\circ} \mathrm{C}$
IR $\left(\mathrm{cm}^{-1}\right) 3051,1456,1435,1390,1155,799,772,743$, 725.

${ }^{1} \mathbf{H}$ NMR $\left(\mathrm{CDCl}_{3}, 300 \mathrm{MHz}, 298 \mathrm{~K}\right): \delta \mathrm{ppm}$ 7.38-7.48 (m, 2H), 7.49 (s, 1H), 7.53-7.58 (m, 3H), 7,69 (d, 1H, $J=7,0$ $\mathrm{Hz})$, 7.87-7.96 (m, 4H), 8.32-8.35 (m, 1H).

${ }^{13} \mathrm{C}$ NMR $\left(\mathrm{CDCl}_{3}, 75 \mathrm{MHz}, 298 \mathrm{~K}\right): \delta$ ppm 122.2, 123.7, 124.2 , 124.4, 124.6, 125.3, 125.9, 126.3, 126.8, 128.5, $128.6,129.0,131.9,132.5,133.9,140.3,140.4,142.3$.

MS (APCI) $m / z=261(\mathrm{M}+\mathrm{H})^{+}$

\section{2-(Benzo $[b]$ thiophen-2-yl)phenol 7h}

Yield : $88 \%$; colorless solid; $\mathrm{mp} 91-94^{\circ} \mathrm{C}$.

IR $\left(\mathrm{cm}^{-1}\right) 3511,3054,1481,1449,1432,1333,1290,1173$, 747.

${ }^{1} \mathbf{H}$ NMR $\left(\mathrm{CDCl}_{3}, 300 \mathrm{MHz}, 298 \mathrm{~K}\right): \delta \mathrm{ppm} 5.63(\mathrm{~s}, 1 \mathrm{H})$, 6.99-7.05 (m, 2H), 7.27-7.43 (m, 3H), $7.40(\mathrm{dd}, 1 \mathrm{H}, J=7.6$ $\mathrm{Hz}, J=1.6 \mathrm{~Hz}), 7.56$ (s, 1H), 7.81-7.89 (m, 2H).

${ }^{13} \mathrm{C}$ NMR $\left.\left(\mathrm{CDCl}_{3}, 75 \mathrm{MHz}, 298 \mathrm{~K}\right): \delta \mathrm{ppm}\right): 116.5,121.0$, $121.2,122.3,123.0,123.8,124.7,124.8,130.0,130.4$, $139.4,140.0,140.4,152.9$.

MS (APCI+) $m / z=227.0(\mathrm{M}+\mathrm{H})^{+}$

Anal. Calcd for $\mathrm{C}_{14} \mathrm{H}_{10} \mathrm{OS}$ : C, 74.31; H, 4.45. Found C, $74.01 ; \mathrm{H}, 4.22$.

\section{3-(2-Methoxyphenyl)-1 $H$-isochromen-1-one $7 \mathbf{i}^{23}$}

Yield : 95\%; white solid; mp $122-124^{\circ} \mathrm{C}$.

IR $\left(\mathrm{cm}^{-1}\right)$ 1724, 1625, 1493, 1225, 1021, 754.

${ }^{1} \mathbf{H}$ NMR $\left(\mathrm{CDCl}_{3}, 300 \mathrm{MHz}, 298 \mathrm{~K}\right): \delta \mathrm{ppm} 3.96$ (s, 3H), $7.00(\mathrm{~d}, 1 \mathrm{H}, J=8.3 \mathrm{~Hz}), 7.07(\mathrm{t}, 1 \mathrm{H}, J=8.0 \mathrm{~Hz}), 7.36-7.40$ $(\mathrm{m}, 2 \mathrm{H}), 7.45-7.51(\mathrm{~m}, 2 \mathrm{H}), 7.70(\mathrm{t}, 1 \mathrm{H}, J=8.3 \mathrm{~Hz}), 7.97$ (dd, $1 \mathrm{H}, J=7.9, J=1.7 \mathrm{~Hz}), 8.30(\mathrm{~d}, 1 \mathrm{H}, J=7.9 \mathrm{~Hz})$.

${ }^{13} \mathrm{C}$ NMR $\left(\mathrm{CDCl}_{3}, 75 \mathrm{MHz}, 298 \mathrm{~K}\right): \delta \mathrm{ppm} 55.8,107.1$, $111.5,120.8,120.9,121.0,126.4,128.1,129.0,129.5$, $130.9,134.8,138.2,150.6,157.4,162.8$.

MS (APCI) $m / z=253(\mathrm{M}+\mathrm{H})^{+}$

\section{2-(2-Methylprop-1-enyl)benzofuran $10 a^{35}$}

Yield : $72 \%$; colorless solid; $\mathrm{mp} 44-48^{\circ} \mathrm{C}$.

IR $\left(\mathrm{cm}^{-1}\right)$ 2929, 1453, 1196, 1055, 847, 790, 748.

${ }^{1} \mathbf{H}$ NMR $\left(\mathrm{CDCl}_{3}, 300 \mathrm{MHz}, 298 \mathrm{~K}\right): \delta \mathrm{ppm} 1.98(\mathrm{~s}, 3 \mathrm{H})$, $2.13(\mathrm{~s}, 3 \mathrm{H}), 6.20(\mathrm{~s}, 1 \mathrm{H}), 6.51(\mathrm{~s}, 1 \mathrm{H}), 7.17-7.25(\mathrm{~m}, 2 \mathrm{H})$, $7.43(\mathrm{~d}, 1 \mathrm{H}, J=7.5 \mathrm{~Hz}), 7.43(\mathrm{~d}, 1 \mathrm{H}, J=7.5 \mathrm{~Hz})$.

${ }^{13} \mathrm{C}$ NMR $\left(\mathrm{CDCl}_{3}, 75 \mathrm{MHz}, 298 \mathrm{~K}\right)$ : $\delta \mathrm{ppm} 20.7,27.5$, $103.8,110.9,114.7,120.5,122.7,123.7,129.2,139.7$, 154.2, 155.8 .

MS (APCI+) $m / z=173.0(\mathrm{M}+\mathrm{H})^{+}$

(E)-2-(Hex-1-enyl)benzofuran 10b

Yield : $47 \%$.

${ }^{1} \mathbf{H}$ NMR $\left(\mathrm{CDCl}_{3}, 300 \mathrm{MHz}, 298 \mathrm{~K}\right)$ : $\delta$ ppm 0.93-0.98 (m, $3 \mathrm{H}), 1.37-1.53(\mathrm{~m}, 4 \mathrm{H}), 2.27(\mathrm{q}, 2 \mathrm{H}, J=6.8 \mathrm{~Hz}), 6.33(\mathrm{~d}$, $1 \mathrm{H}, J=16.0 \mathrm{~Hz}), 6.47-6.60(\mathrm{~m}, 2 \mathrm{H}), 7.15-7.26(\mathrm{~m}, 2 \mathrm{H})$, 7.41-7.50 (m, 2H).

${ }^{13} \mathrm{C}$ NMR $\left(\mathrm{CDCl}_{3}, 75 \mathrm{MHz}, 298 \mathrm{~K}\right): \delta \mathrm{ppm} \mathrm{14.1,} \mathrm{22.4,}$ $31.3,32.8,102.7,110.9,118.7,120.7,122.8,124.1,129.3$, 134.1, 154.7, 155.4 .

Anal. Calcd for $\mathrm{C}_{14} \mathrm{H}_{16} \mathrm{O}$ : C, 83.96; H, 8.05. Found C, 83.66; H, 7.82. 
(E)-2-Styrylbenzofuran $10 \mathbf{c}^{30}$

Yield : 73\%; colorless solid; $124-126 \mathrm{mp}{ }^{\circ} \mathrm{C}$.

IR $\left(\mathrm{cm}^{-1}\right)$ 3057, 1450, 1256, 958, 907, 804, 738, 691.

${ }^{1} \mathbf{H}$ NMR $\left(\mathrm{CDCl}_{3}, 300 \mathrm{MHz}, 298 \mathrm{~K}\right): \delta \mathrm{ppm} 6.70(\mathrm{~s}, 1 \mathrm{H})$, $7.03(\mathrm{~d}, 1 \mathrm{H}, J=16.2 \mathrm{~Hz}), 7.20-7.42(\mathrm{~m}, 6 \mathrm{H}), 7.50(\mathrm{~d}, 1 \mathrm{H}, J$ $=8.0 \mathrm{~Hz}), 7.55(\mathrm{~d}, 3 \mathrm{H}, J=7.4 \mathrm{~Hz})$.

${ }^{13} \mathrm{C}$ NMR $\left(\mathrm{CDCl}_{3}, 75 \mathrm{MHz}, 298 \mathrm{~K}\right): \delta \mathrm{ppm} 105.3,111.0$, 116.6, 121.0, 123.0, 124.8, 126.8 (2), 128.3, 128.9, 129.3, $130.4,136.7,155.0,155.2$.

MS (APCI+) $m / z=221.0(\mathrm{M}+\mathrm{H})^{+}$

(E)-2-(4-Methoxystyryl)benzofuran 10d

Yield : $75 \%$; colorless solid; $\mathrm{mp} 143^{\circ} \mathrm{C}$.

IR $\left(\mathrm{cm}^{-1}\right)$ 2996, 1600, 1506, 1450, 1238, 1176, 1025, 965, 944, 826, 816, 743 .

${ }^{1} \mathbf{H}$ NMR $\left(\mathrm{CDCl}_{3}, 300 \mathrm{MHz}, 298 \mathrm{~K}\right): \delta \mathrm{ppm} 3.85$ (s, 3H), $6.63(\mathrm{~s}, 1 \mathrm{H}), 6.86-6.94(\mathrm{~m}, 3 \mathrm{H}), 7.18-7.32$ (m, 3H), 7.47$7.54(\mathrm{~m}, 4 \mathrm{H})$

${ }^{13} \mathrm{C}$ NMR $\left(\mathrm{CDCl}_{3}, 75 \mathrm{MHz}, 298 \mathrm{~K}\right): \delta$ ppm 55.5, 104.4, $110.9,114.4$ (2), $114.5,120.8,122.9,124.4,128.2$ (2), $129.4,129.5,130.1,154.9,155.6,159.9$.

MS (APCI+) $m / z=251.0(\mathrm{M}+\mathrm{H})^{+}$

Anal. Calcd for $\mathrm{C}_{17} \mathrm{H}_{14} \mathrm{O}_{2}$ : C, 81.58; H, 5.64. Found C, 81.44; H, 5.52 .

\section{(E)-2-Styrylbenzo[b]thiophene $10 \mathrm{e}^{36}$}

Yield : $78 \%$; beige solid; $\mathrm{mp} 198^{\circ} \mathrm{C}$.

IR $\left(\mathrm{cm}^{-1}\right)$ 2926, 1447, 1432, 947, 818, 739, 725, 690 .

${ }^{1} \mathbf{H}$ NMR $\left(\mathrm{CDCl}_{3}, 300 \mathrm{MHz}, 298 \mathrm{~K}\right): \delta \mathrm{ppm} 7.01(\mathrm{~d}, 1 \mathrm{H}, J$ $=16.0 \mathrm{~Hz}), 7.26-7.40(\mathrm{~m}, 7 \mathrm{H}), 7.52(\mathrm{~d}, 2 \mathrm{H}, J=7.4 \mathrm{~Hz})$, $7.70(\mathrm{~m}, 1 \mathrm{H}), 7.78(\mathrm{~m}, 1 \mathrm{H})$.

${ }^{13} \mathrm{C}$ NMR $\left(\mathrm{CDCl}_{3}, 75 \mathrm{MHz}, 298 \mathrm{~K}\right): \delta \mathrm{ppm} 122.4,122.5$, $123.4,123.6,124.7,124.9,126.7$ (2), 128.2, 128.9 (2), 131.0, 136.8, 139.1, 140.4, 143.1 .

MS (APCI+) $m / z=237.0(\mathrm{M}+\mathrm{H})^{+}$

\section{2,2'-Bibenzofuran 11a}

Yield : 58\%; colorless solid; mp $177-178^{\circ} \mathrm{C}$.

IR $\left(\mathrm{cm}^{-1}\right) 1439,1255,1172,1048,875,814,748,611$.

${ }^{1} \mathbf{H}$ NMR $\left(\mathrm{CDCl}_{3}, 300 \mathrm{MHz}, 298 \mathrm{~K}\right)$ : $\delta$ ppm 6.92-6.96 (m, $2 \mathrm{H}), 7.03(\mathrm{~m}, 1 \mathrm{H}), 7.07(\mathrm{~m}, 1 \mathrm{H}), 7.18-7.27(\mathrm{~m}, 3 \mathrm{H}), 7.46$ $(\mathrm{m}, 1 \mathrm{H}), 7.54(\mathrm{~m}, 1 \mathrm{H}), 7.65(\mathrm{~m}, 1 \mathrm{H})$.

${ }^{13} \mathbf{C}$ NMR $\left(\mathrm{CDCl}_{3}, 75 \mathrm{MHz}, 298 \mathrm{~K}\right): \delta \mathrm{ppm} 103.8,111.4$, $121.5,123.5,125.2,128.7,147.8,155.2$.

MS (APCI+) $\mathrm{m} / \mathrm{z}=235.0(\mathrm{M}+\mathrm{H})^{+}$.

Anal. Calcd for $\mathrm{C}_{16} \mathrm{H}_{10} \mathrm{O}_{2}$ : C, 82.04; $\mathrm{H}, 4.30$. Found $\mathrm{C}$, 81.77; H, 4.12.

\section{2-(Benzo[b]thiophen-2-yl)benzofuran 11b}

Yield : $76 \%$; colorless solid; mp $207-209^{\circ} \mathrm{C}$.

IR $\left(\mathrm{cm}^{-1}\right)$ 3055, 1426, 1202, 988, 932, 878, 827, 799, 737, 625.

${ }^{1} \mathbf{H}$ NMR $\left(\mathrm{CDCl}_{3}, 300 \mathrm{MHz}, 298 \mathrm{~K}\right): \delta \mathrm{ppm} 7.03(\mathrm{~s}, 1 \mathrm{H})$, 7.29-7.44 (m, 4H), $7.57(\mathrm{~d}, 1 \mathrm{H}, J=8.0 \mathrm{~Hz}), 7.62(\mathrm{~d}, 1 \mathrm{H}, J$ $=7.4 \mathrm{~Hz}), 7.76(\mathrm{~s}, 1 \mathrm{H}), 7.85(\mathrm{~m}, 2 \mathrm{H})$.
${ }^{13} \mathrm{C}$ NMR $\left(\mathrm{CDCl}_{3}, 75 \mathrm{MHz}, 298 \mathrm{~K}\right): \delta \mathrm{ppm} 103.4,111.3$, $121.1,121.2,122.4,123.4,124.1,124.9,125.0,125.1$, 129.0, 133.0, 139.7, 140.2, 151.1, 155.5 .

MS (APCI+) $m / z=251.0(\mathrm{M}+\mathrm{H})^{+}$

Anal. Calcd for $\mathrm{C}_{16} \mathrm{H}_{10} \mathrm{OS}$ : C, 76.77; H, 4.03. Found C, 76.44; H, 3.80.

\section{2-(Benzofuran-2-yl)-1-(4-methoxyphenyl)ethanone 11c} Yield : $94 \%$; colorless solid; $\mathrm{mp} 104-106^{\circ} \mathrm{C}$.

IR $\left(\mathrm{cm}^{-1}\right)$ 2936, 1678, 1454, 1251, 1219, 1170, 751.

${ }^{1} \mathbf{H}$ NMR $\left(\mathrm{CDCl}_{3}, 300 \mathrm{MHz}, 298 \mathrm{~K}\right): \delta \mathrm{ppm} 3.87$ (s, 3H), $4.40(\mathrm{~s}, 2 \mathrm{H}), 6.62(\mathrm{~s}, 1 \mathrm{H}), 6.95(\mathrm{~d}, 2 \mathrm{H}, J=8.7 \mathrm{~Hz}), 7.16-$ $7.26(\mathrm{~m}, 2 \mathrm{H}), 7.43(\mathrm{~d}, 1 \mathrm{H}), 7.50(\mathrm{~d}, 1 \mathrm{H}), 8.04(\mathrm{~d}, 2 \mathrm{H}, J=$ $8.7 \mathrm{~Hz})$.

${ }^{13} \mathrm{C}$ NMR $\left(\mathrm{CDCl}_{3}, 75 \mathrm{MHz}, 298 \mathrm{~K}\right): \delta \mathrm{ppm} 38.8,55.7$, $105.3,111.2$, 114.1 (2), 120.8, 122.8, 123.9, 128.8, 129.3, 131.2 (2), 152.0, 155.1, 164.0, 193.1.

MS (APCI+) $m / z=289.0(\mathrm{M}+\mathrm{Na})^{+}$

Anal. Calcd for $\mathrm{C}_{17} \mathrm{H}_{14} \mathrm{O}_{3}$ : C, 76.68; H, 5.30. Found C, 76.39; H, 4.99.

\section{2-(Naphthalen-2-ylethynyl)benzofuran 11d}

Yield : $28 \%$; beige solid; $\mathrm{mp} 136-137^{\circ} \mathrm{C}$.

IR $\left(\mathrm{cm}^{-1}\right)$ 3058, 2934, 1448, 1258, 1165, 906, 819, 739.

${ }^{1} \mathbf{H}$ NMR $\left(\mathrm{CDCl}_{3}, 300 \mathrm{MHz}, 298 \mathrm{~K}\right)$ : $\delta$ ppm $7.07(\mathrm{~d}, 1 \mathrm{H}, J$ $=0.7 \mathrm{~Hz}), 7.27-7.32(\mathrm{~m}, 1 \mathrm{H}), 7.38(\mathrm{~m}, 1 \mathrm{H}), 7.51-7.57(\mathrm{~m}$, $3 \mathrm{H})$, 7.60-7.66 (m, 2H), 7.85-7.88 (m, 3H), 8.15 (s, 1H).

${ }^{13} \mathrm{C}$ NMR $\left(\mathrm{CDCl}_{3}, 75 \mathrm{MHz}, 298 \mathrm{~K}\right): \delta \mathrm{ppm} 80.2$, 95.7, $111.4,111.8,119.3,121.4,123.5,125.8,126.9,127.3$, $128.0,128.1,128.4,132.1,133.1,133.3,139.0,150.5$, 155.1 .

MS (APCI+) $m / z=269.0(\mathrm{M}+\mathrm{H})^{+}$

Anal. Calcd for $\mathrm{C}_{20} \mathrm{H}_{12} \mathrm{O}$ : C, 89.53; H, 4.51. Found $\mathrm{C}$, 89.11; H, 4.32.

\section{References}

1 For an excellent review, see: Hintermann, L.; Labonne, A. Synthesis 2007, 1121.

2 Kucherov, M. Chem. Ber. 1881, 14, 1540.

3 (a) Marion, N.; Ramón, R. S.; Nolan, S. P. J. Am. Chem. Soc. 2009, 131, 448. (b) Mizushima, E.; Sato, K.; Hayashi, T.; Takana, M. Angew; Chem., Int. Ed; 2002, 41, 4563. (c) Casado, R.; Contel, M.; Laguna, M.; Romero, P.; Sanz, S. J. Am. Chem. Soc. 2003, 125,11925 .

4 (a) Tokunaga, M.; Wakatsuki, Y. Angew. Chem. Int. Ed. 1998, 37, 2867. (b) Chen, Y.; Valentini, M.; Pregosin, P. S.; Albinati, A. Inorg.Chim. Acta 2002, 327, 4. (c) Alvarez, P.; Gimeno, J.; Lastra, E.; GarcíaGranda, S.; Van der Maelen, J. F.; Bassetti, M. Organometallics 2001, 20, 3762.

5 (a) Hirabayashi, T.; Okimoto, Y.; Saito, A.; Morita, M.; Sakaguchi, S.; Ishii, Y. Tetrahedron Lett. 2006, 62, 2231. (b) Chin, C. S.; Chang, W.-T.; Yang, S.; Joo, K.-S. Bull. Korean Chem. Soc. 1997, 18, 324. (c) 
Kanemitsu, H.; Uehara, K.; Fukuzumi, S.; Ogo, S. J. Am. Chem. Soc. 2008, 130, 17141.

6 (a) James, B. R.; Rempel, G. L. J. Am. Chem. Soc. 1969, 91, 863. (b) Blum, J.; Huminer, H.; Alper, H. J. Mol. Catal. 1992, 75, 153.

7 (a) Baidossi, W.; Lahav, M.; Blum, J. J. Org. Chem. 1997, 62, 669. (b) Israelsohn, O.; Vollhardt, K. P. C.; Blum, J. J.Mol. Catal. 2002, 184, 1. (c) Hartman, J. W.; Sperry, L. Tetrahedron Lett. 2004, 45, 3787.

$8 \quad$ Liu, B.; De Brabander, J. K. Org. Lett. 2006, 8, 4907.

9 (a) Boll, M.; Schink, B.; Messerschmidt, A.; Kroneck, P. M. H. Biol. Chem. 2005, 386, 999. (b) Alipui, O. D.; Zhang, D.; Schulz, H. Biochem. Biophys. Res. Commun. 2002, 292, 1171. (c) Wang, S. C.; Person, M. D.; Johnson, W. H.; Whitman, C. P. Biochemistry 2003, 42, 8762 .

10 Tsuchimoto, T.; Joya, T.; Shirakawa, E.; Kawakami, Y. Synlett, 2000, 1777.

11 Allen, A. D.; Chiang, Y.; Kresge, A. J.; Tidwell, T. T. J. Org. Chem. 1982, 47, 775 and references cited therein.

12 Menash, N.; Shvo, Y. J. Org. Chem. 1993, 58, 7434.

13 (a) Alami, M.; Ferri, F. Synlett 1996, 755; (b) Liron, F.; Le Garrec, P.; Alami, M. Synlett 1999, 246; (c) Alami, M.; Liron, F.; Gervais, M.; Peyrat, J.-F.; Brion, J.-D. Angew. Chem., Int. Ed. 2002, 41, 1578; (d) Hamze, A.; Provot, O.; Alami, M.; Brion, J.-D. Org. Lett. 2005, 7, 5625; (e) Hamze, A.; Provot, O.; Brion, J.-D.; Alami, M. Synthesis 2007, 2025; (f) Hamze, A.; Provot, O.; Brion, J.-D.; Alami, M. J. Org. Chem. 2007, 72, 3868; (g) Giraud, A.; Provot, O.; Hamze, A.; Brion, J.-D.; Alami, M. Tetrahedron Lett. 2008, 49, 1107.

14 Kwatra, M. M.; Simon, D. Z.; Salvador, R. L.; Cooper, P. D. J. Med. Chem. 1978, 21, 253.

15 Olivi, N.; Thomas, E.; Peyrat, J.-F.; Alami, M.; Brion, J.-D. Synlett 2004, 2175.

16 (a) Sonogashira, K.; Tohda, Y.; Hagihara, N. Tetrahedron Lett. 1975, 4467. (b) Alami, M.; Ferri, F.; Linstrumelle, G. Tetrahedron Lett. 1993, 34, 6403.

17 For a review see: Swaminathan, S.; Narayanan, K. V. Chem. Rev. 1971, 71, 429.

18 (a) Tzalis, D.; Koradin, C.; Knochel, P. Tetrahedron Lett. 1999, 40, 6193. (b) Hartman, J. W.; Sperry, L. Tetrahedron Lett. 2004, 45, 3787. (c) For a review, see: Alonso, F.; Beletskaya, I. P.; Yus, M. Chem. Rev. 2004, 104, 3079.

19 (a) Thierney, J.P.; Lidström, P. Microwave Assisted Organic Synthesis; Blackwell Publishing Ldt.; 2005. (b) Caddick, S. Tetrahedron 1995, 51, 10403. (c) Perreux, L.; Loupy, A. Tetrahedron 2001, 57, 9199. (d) Lisström, P.; Thierney, J.; Wathey, B.; Westman, J. Tetrahedron 2001, 57, 9225. (e) Bekaert, A.; Provot, O.; Rasolojaona, O.; Peyrat, J.-F.; Alami, M.; Brion, J.-D. Tetrahedron Lett. 2005, 46, 4187. (f) Le
Bras, G.; Provot, O.; Bekaert, A.; Peyrat, J.-F.; Alami, M.; Brion, J.-D. Synthesis 2006, 1537. (g) L'Hermite, N.; Giraud, A.; Provot, O.; Peyrat, J.-F.; Alami, M.; Brion, J.-D. Tetrahedron 2006, 62, 11994. (h) Giraud, A.; Provot, O.; Peyrat, J.-F.; Alami, M.; Brion, J.-D. Tetrahedron 2006, 62, 7667. (i) Le Bras, G.; Provot, O.; Peyrat, J.-F.; Alami, M.; Brion, J.-D. Tetrahedron Lett. 2006, 47, 5497. (j) Le Bras, G.; Radanyi, C.; Peyrat, J.-F.; Brion, J.-D.; Alami, M.; Marsaud, V.; Stella, B.; Renoir, J.-M. J. Med. Chem. 2007, 50, 6189.

20 (a) Pettit, G.R.; Singh, S.B.; Niven, M.L.; Hamel, E.; Schmidt, J.M. J. Nat. Prod. 1987, 50, 119. (b) Provot, O.; Giraud, A.; Peyrat, J.-F.; Alami, M.; Brion, J.-D. Tetrahedron Lett. 2005, 46, 8547. (c) Mousset, C.; Giraud, A. Provot, O.; Hamze, A.; Bignon, J.; Liu, J.M.; Thoret, S.; Dubois, J.; Brion, J.-D.; Alami, M. Bioorg. Med. Chem. Lett. 2008, 18, 3266. (d) Giraud, A.; Provot, O.; Hamze, A.; Brion, A.; Alami, M. Tetrahedron Lett. 2008, 49, 1107. Hamze, A.; (e) Hamze, A.; Giraud, A. Messaoudi, M.; Provot, O.; Peyrat, J.-F.; Bignon, J. Liu, J.-M.; WdzieczakBakala, J.; Thoret, S.; Dubois, J.; Brion, J.-D.; Alami, M. ChemMedChem 2009, 4, 1912. (f) Messaoudi, S.; Tréguier, B.; Hamze, A.; Provot, O.; Peyrat, J.-F.; Rodrigo De Losada, J.; Liu, J.-M.; Bignon, J.; Wdzieczak-Bakala, J.; Thoret, S.; Dubois, J.; Brion, J.-D., Alami, M. J. Med. Chem., 2009, 524538.

21 Jacubert, M.; Hamze, A.; Provot, O.; Peyrat, J.-F.; Brion, J.-D.; Alami, M. Tetrahedron Lett. 2009, 50, 3588 .

22 For benzofuran derivatives, see for example: (a) Donnelly, D. M. X.; Meegan, M. J. Comprehensive Heterocyclic Chemistry; Katritzky, A. R., Ed.; Pergamon Press: New York, 1984; vol. 4. (b) Bakunov, S.; Bakunova, S.; Wenzler, T.; Barszcz, T.; Werbovetz, K.; Brun, R.; Tidwell, R. J. Med. Chem. 2008, 51, 6927. (c) McCallion, G. D. Curr. Org. Chem. 1999, 3, 67. For benzothiphene derivatives see for example: (d) Palkowitz, A. D.; Glasebrook, A. L.; Thrascher, K. J.; Hauser, K. L.; Short, L. L.; Phillips, D. L.; Muehl, B. S.; Sato, M.; Shetler, P. K.; Cullinan, G. J.; Pell, T. R.; Bryant, H. U. J. Med. Chem. 1997, 40, 1407. (e) Chen, Z.; Mocharla, V. P.; Farmer, J. M.; Pettit, G. R.; Hamel, E.; Pinney, K. G. J. Org. Chem. 2000, 65, 8811. (f) Fournier Dit Chabert, J. F.; Joucla, L.; David, E.; Lemaire, M. Tetrahedron 2004, $60,3221$.

23 Le Bras, G.; Hamze, A.; Messaoudi, S.; Provot, O.; Le Calvez, P.-B.; Brion, J.-D.; Alami, M. Synthesis 2008, 1607.

24 Seck, M.; Franck, X.; Hocquemiller, R.; Figadère, B.; Peyrat, J.-F.; Provot, O.; Brion, J.-D.; Alami, M. Tetrahedron Lett. 2004, 45, 1881. 
25 (a) Ramiandrasoa, P.; Bréhon, B.; Thivet, A.; Alami, M.; Cahiez, G. Tetrahedron Lett. 1997, 38, 2447. (b) Peyrat, J.-F.; Thomas, E.; L'Hermite, N.; Alami, M.; Brion, J.-D. Tetrahedron Lett. 2003, 44, 6703.

Alami, M.; Ferri, F. Tetrahedron Lett. 1996, 37, 2763.

27 (a) Byun, J. H.; Kim, H.; Kim, Y.; Mook-Jung, I.; Kim, D. J.; Lee, W. L.; Yoo, K. H. Bioorg. Med. Chem. Lett. 2008, 18, 5591. (b) Hayakawa, I.; Shioya, R.; Agatsuma, T.; Furukawa, H.; Naruto, S.; Sugano, Y. Bioorg. Med. Chem. Lett. 2004, 14, 455. (c) Yoo, H.; Lee, L. Y.; Park, J. H.; Chung, B. Y.; Lee, Y. S. Farmaco, 2003, 58, 1243.

Bietti, M.; Capone, A. J. Org. Chem. 2008, 73, 618.

Atkinson, R.S. J. Chem. Soc. C 1971, 4, 784.

Commercially available

31 Park, J.Y.; Ullapu, P.R.; Choo, H.; Lee, J.K.; Min, S.J.; Pae, A.N.; Kim, Y.; Baek, D.-J.; Cho, Y.S. Eur. J. Org. Chem. 2008, 5461.

32 Duan, X.-F.; Zeng, J.; Zhang, Z.-B.; Zi, G.-F. J. Org. Chem. 2007, 72, 10283.

33 Clerici, A.; Porta, O. J. Org. Chem. 1990, 55, 1240.

34 Ohta, A.; Akita, Y.; Ohkuwa, T.; Chiba, M.; Fukunaga, R. Heterocycles 1990, 31, 1951.

35 Macleod, C.; McKiernan, G.J.; Guthrie, E.J.; Farrugia, L.J.; Hamprecht, D.W.; Macritchie, J.; Hartley, R.C. J. Org. Chem. 2003, 68, 387.

36 Bryan, C.S.; Braunger, J.A.; Lautens, M. Angew. Chem. Int. Ed. 2009, 48, 7064. 